\title{
FECUNDIDAD Y ESCOLARIDAD EN LA CIUDAD DE MÉXICO
}

\author{
Jorge H. Zambrano Lupi \\ Universidad de los Andes \\ Mérida, Venezuela
}

\section{INTRODUCCIÓN}

BASTANTE CONOCIDA es la diversidad de orientaciones bajo las cuales ha sido expuesta la bibliografía sobre la fecundidad: análisis de naturaleza principalmente cuantitativa; investigaciones globales presentadas, entre otras, en términos de explosión demográfica, control familiar, planificación familiar, tamaño de la familia, métodos y uso de anticonceptivos, transición demográfica, diferenciales de fecundidad, modernización, marcos teóricos, reseñas críticas, etc., bibliografía que en cada uno de los casos, ha seguido el curso de los lineamientos que han dado lugar a la misma.

El presente estudio tiene por objeto analizar la relación entre nivel de fecundidad y grado de escolaridad en la ciudad de México. Se orienta con la óptica de los estudios de fecundidad diferencial. En ellos, por lo regular, se trata de establecer una relación entre dos variables, una de ellas, la fecundidad, se considera variable dependiente, y otra cualquiera, en este caso la escolaridad, se considera como variable independiente.

A este tipo de análisis, al igual que la mayoría de los señalados, se le ha criticado su pobreza explicativa, aun cuando se reconocen sus méritos de tipo descriptivo. No obstante, a nuestro juicio, los trabajos de fecundidad diferencial, unos con menor éxito, otros con mayor acierto, han aportado su contribución al conocimiento del estudio de la fecundidad, sobre todo en su relación con el contexto socioeconómico.

Con la intención de apartarnos de la línea en extremo descriptiva que ha caracterizado a buena parte de los análisis de fecundidad diferencial, se tratará no sólo de establecer las diferencias comparativas que pueden darse entre el nivel de la fecundidad y el grado de escolaridad, sino también de encontrar la explicación del porqué de las mismas. En ese sentido, se evita el análisis de la relación fecundidad-escolaridad per se, es decir, no se admite como implicación necesaria del tipo causa-efecto; más bien se tiene en cuenta que, así como la variable fecundidad obedece a múltiples efectos de la totalidad social en la cual el individuo desempeña su existencia, el grado de escolaridad alcanzado está a su vez interrelacionado y deter- 
minado por una serie de variables. Entre ellas se ha detectado que la situación de clase social pesa de manera importante, situación que a su vez está en estrecha dependencia con factores tales como la ocupación, el nivel de ingresos, el lugar de residencia, etc.

En última instancia a lo que se pretende llegar es a precisar qué tanto la reproducción de la población y su nivel de escolaridad en un momento dado, no pueden ser considerados como variables autónomas, sino como variables dependientes, que reflejan el juego de factores sociales más amplios que las superan, engloban y determinan.

La razón de haber escogido el diferencial fecundidad-escolaridad, radica en que, como muy bien lo afirman, entre otros autores, Miró y Mertens $(1,1969$, p. 14) "ninguna otra variable socioeconómica evidencia una relación negativa tan nítida respecto a la fecundidad como la educación, observándose dicha relación, tanto en las áreas urbanas como en las áreas rurales-semiurbanas".

Para el análisis entre el nivel de fecundidad, y el grado de escolaridad en la ciudad de México, se utiliza la información de la encuesta sobre $M i$ gración interna, estructura ocupacional y movilidad social, efectuada durante 1970, en el área metropolitana de la ciudad de México (véase, Muñoz, Oliveira y Stern, 2, 1977).

En primer lugar se caracteriza de manera breve la situación educativa en el país y en la ciudad de México. A continuación se postula la hipótesis de trabajo y se esbozan algunas razones para justificar la formulación de la misma. Se examina después la relación entre el nivel de fecundidad y el grado de escolaridad en la ciudad de México, primero en función de la edad de las madres y luego incorporando consideraciones sobre la posición social de sus compañeros. Por último, se establecen las principales conclusiones. Se agrega al cuerpo de trabajo un anexo sobre los ejercicios de estandarización realizados y la descripción de las principales características de la encuesta utilizada en la elaboración del trabajo.

\section{Algunas CARACTERÍsticas DE LA SITUACión EDUCATIVA EN LA CIUDAD DE MÉXICO}

En esta parte se hace un esbozo de las principales características del sistema educativo en la ciudad de México durante los últimos cincuenta años. Al mismo tiempo, se establecen diferencias con el sistema educativo a nivel nacional. ${ }^{1}$

1 Se ha considerado el período 1921-1970, debido a que el análisis se hará a través de la información de encuestas efectuadas durante 1970, sobre mujeres que se encontraban dentro de las edades reproductivas (entre los 15 y 50 años de edad). Como puede observarse, las mujeres que para 1970 tenían 50 años de edad, habían nacido en el año 1920, pudiendo afirmarse que su educación primaria comenzó a finales de los años veintes. 
Es conveniente mencionar algunos de los problemas que dificultan el análisis de la situación educativa del país: la calidad y la cantidad de la información censal y estadística varían de manera sustancial entre un año y otro, se operan cambios de conceptos y de clasificaciones, reemplazo de unos cuadros por otros, introducción de nuevas tabulaciones, etc., razones que impiden un análisis completo y obligan a generalizaciones básicas que sólo brindan un panorama global al respecto.

La información que se presenta a continuación permite apreciar algunas de las características sobresalientes del sistema educativo mexicano. El cuadro 1 muestra una visión global de la situación educativa y el 2 proporciona una idea de la expansión del sistema educativo a través de la demanda potencial y de la matrícula escolar primaria.

\section{Cuadro 1}

Número de aNAlfabetas de la POBlación tOtal del País Y DEL Distrito Federal, 1921-1970

\begin{tabular}{|c|c|c|c|c|c|c|c|c|c|c|c|c|c|}
\hline \multirow{2}{*}{\multicolumn{2}{|c|}{ Años }} & \multicolumn{4}{|c|}{ Total } & \multicolumn{4}{|c|}{ Hombres } & \multicolumn{4}{|c|}{ Kujeres } \\
\hline & & \multicolumn{3}{|c|}{ Número } & \multirow{2}{*}{$\overline{5}$} & \multicolumn{3}{|c|}{ Namero } & \multirow[t]{2}{*}{$\frac{9}{9}$} & \multicolumn{3}{|c|}{ Húmero } & \multirow[t]{2}{*}{$\overline{8}$} \\
\hline & & & & & & & & & & & & & \\
\hline $\begin{array}{l}\text { Kéxico } \\
\text { Distrito }\end{array}$ & Federal & 6 & $\begin{array}{l}973 \\
192\end{array}$ & $\begin{array}{l}855 \\
645\end{array}$ & $\begin{array}{l}66.2 \\
26.9\end{array}$ & 3 & $\begin{array}{r}195 \\
67\end{array}$ & $\begin{array}{l}342 \\
499\end{array}$ & $\begin{array}{l}.63 .0 \\
21.4\end{array}$ & 3 & $\begin{array}{l}778 \\
125\end{array}$ & $\begin{array}{l}013 \\
146\end{array}$ & $\begin{array}{l}69.2 \\
31.2\end{array}$ \\
\hline 1930 & & & & & 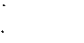 & & & & & & . & & \\
\hline $\begin{array}{l}\text { Mexico } \\
\text { Distrito }\end{array}$ & Federal & 6 & $\begin{array}{l}962 \\
215\end{array}$ & $\begin{array}{l}517 \\
597\end{array}$ & $\begin{array}{l}59.3 \\
23.1\end{array}$ & 3 & $\begin{array}{r}100 \\
62\end{array}$ & $\begin{array}{l}945 \\
267\end{array}$ & $\begin{array}{l}54.6 \\
15.2\end{array}$ & 3 & $\begin{array}{l}861 \\
153\end{array}$ & $\begin{array}{l}571 \\
330\end{array}$ & $\begin{array}{l}63.6 \\
29.3\end{array}$ \\
\hline 1940 & & & & & & & & & & & & & \\
\hline $\begin{array}{l}\text { Mexico } \\
\text { Uistrito }\end{array}$ & Eederal & 7 & $\begin{array}{l}198 \\
243\end{array}$ & $\begin{array}{l}756 \\
468\end{array}$ & $\begin{array}{l}51.6 \\
18.1\end{array}$ & 3 & $\begin{array}{r}2365 \\
69\end{array}$ & $\begin{array}{l}430 \\
420\end{array}$ & $\begin{array}{l}47.6 \\
11.6\end{array}$ & 3 & $\begin{array}{l}962 \\
174\end{array}$ & $\begin{array}{l}326 \\
048\end{array}$ & $\begin{array}{l}55.4 \\
23.4\end{array}$ \\
\hline 1950 & & & & & & & & & & & & & \\
\hline $\begin{array}{l}\text { México } \\
\text { Distrito }\end{array}$ & Federal & 8 & $\begin{array}{l}492 \\
461\end{array}$ & $\begin{array}{l}399 \\
857\end{array}$ & $\begin{array}{l}42.5 \\
18.1\end{array}$ & 4 & $\begin{array}{l}019 \\
156\end{array}$ & $\begin{array}{l}171 \\
283\end{array}$ & $\begin{array}{r}39.0 \\
13.4\end{array}$ & 4 & $\begin{array}{l}9232 \\
3055\end{array}$ & $\begin{array}{l}228 \\
574\end{array}$ & $\begin{array}{l}45.8 \\
22.2\end{array}$ \\
\hline 1960 & & & & & & & & & & & & & \\
\hline $\begin{array}{l}\text { México } \\
\text { Distrito }\end{array}$ & Federal & $10 \%$ & $\begin{array}{l}573 \\
653\end{array}$ & $\begin{array}{l}163 \\
104\end{array}$ & $\begin{array}{l}37.8 \\
16.6\end{array}$ & 4 & $\begin{array}{l}783 \\
224\end{array}$ & $\begin{array}{l}709 \\
607\end{array}$ & $\begin{array}{l}34.4 \\
12.1\end{array}$ & 5 & $\begin{array}{l}789 \\
428\end{array}$ & $\begin{array}{l}454 \\
497\end{array}$ & $\begin{array}{l}41.1 \\
20.6\end{array}$ \\
\hline 1970 & & & & & & & & & & & & & \\
\hline $\begin{array}{l}\text { México } \\
\text { Distrito }\end{array}$ & Eederal & 7 & $\begin{array}{l}677 \\
441\end{array}$ & $\begin{array}{l}073 \\
615\end{array}$ & $\begin{array}{r}23.7 \\
9.1\end{array}$ & 3 & $\begin{array}{l}277 \\
130\end{array}$ & $\begin{array}{l}834 \\
448\end{array}$ & $\begin{array}{r}20.8 \\
5.7\end{array}$ & 4 & $\begin{array}{ll}3992 \\
311 & 1\end{array}$ & $\begin{array}{l}239 \\
167\end{array}$ & $\begin{array}{l}26.9 \\
1.2 .1\end{array}$ \\
\hline
\end{tabular}

Fuente: Elaborado a partir de los cuadros 17 y 24 del Anuario Estadístico de los Estados Unidos Mexicanos para los años 1930 y 1942; y de los cuadros 6, 19 y 11 del Censo General de Población, Resumen General, para los años 1950, 1960 y 1970.

- Para los años 1921, 1930, 1940 y 1970 la información se refiere a la población de 10 años y más. Para los años 1950 y 1960 se refiere a la población de 6 años y más.

Del cuadro 1 se pueden obtener las siguientes apreciaciones:

a) El analfabetismo, en términos relativos, ha descendido de manera 
notable, tanto en el país como en el Distrito Federal. También se observa que el analfabetismo siempre ha sido menor en el caso del Distrito Federal, apreciándose una diferencia muy sensible por cierto (en 1921 tenía una proporción de analafabetas casi igual a la que el país registró en 1970).

b) Su reducción en términos porcentuales no ha implicado necesariamente que con el transcurso del tiempo haya menos analfabetas. Las cifras absolutas muestran lo contrario, es decir, un aumento a medida que aumentó la población. (La diferencia que se aprecia para 1970, obedece al cambio en la población base utilizada como referencia).

c) Las mujeres se han caracterizado por registrar no sólo valores relativos de analfabetismo superiores a los del sexo masculino, sino también por acusar una disminución más lenta del mismo, características apreciables incluso en el caso del Distrito Federal.

\section{Cuadro 2}

Demanda potencial Y matrícula escolar primaria (valores absolutos Y Relativos) EN México Y EL Distrito Federal

\begin{tabular}{|c|c|c|c|c|c|c|c|c|c|c|c|c|}
\hline \multirow{2}{*}{$\begin{array}{l}\text { Años } \\
1925\end{array}$} & \multirow[t]{2}{*}{. } & \multicolumn{3}{|c|}{$\begin{array}{c}\text { Población } \\
\text { total } \\
\text { (1) }\end{array}$} & \multicolumn{3}{|c|}{$\begin{array}{c}\text { Población } 6-14 \\
\text { (2) }\end{array}$} & \multirow[t]{2}{*}{$(2)^{8} /(1)$} & \multicolumn{3}{|c|}{$\begin{array}{l}\text { Población inscrita } \\
(3)\end{array}$} & \multirow[t]{2}{*}{$\frac{\sqrt[0]{5}}{(3)^{2} /(2)}$} \\
\hline & & & & & & & & & & & & \\
\hline $\begin{array}{l}\text { México } \\
\text { Distrito }\end{array}$ & Federal & & & & 2 & $\begin{array}{l}945^{\circ} \\
147\end{array}$ & $\begin{array}{r}519 \\
547\end{array}$ & & & $\begin{array}{l}946 \\
100\end{array}$ & $\begin{array}{l}271 \\
645\end{array}$ & $\begin{array}{l}32.1 \\
68.2\end{array}$ \\
\hline \multicolumn{13}{|l|}{1927} \\
\hline $\begin{array}{l}\text { México } \\
\text { Distrito }\end{array}$ & Eederal & $\begin{array}{r}14 \\
1\end{array}$ & $\begin{array}{l}859 \\
051\end{array}$ & $\begin{array}{l}905 \\
063\end{array}$ & 3 & $\begin{array}{l}055 \\
171\end{array}$ & $\begin{array}{l}486 \\
273\end{array}$ & $\begin{array}{l}20: 6 \\
16.3\end{array}$ & 1 & $\begin{array}{l}306 \\
154\end{array}$ & $\begin{array}{l}557 \\
573\end{array}$ & $\begin{array}{l}42.8 \\
90.2\end{array}$ \\
\hline \multicolumn{13}{|l|}{1930 로 } \\
\hline $\begin{array}{l}\text { México } \\
\text { Distrito }\end{array}$ & Federal & $\begin{array}{r}16 \\
1\end{array}$ & $\begin{array}{l}552 \\
229\end{array}$ & $\begin{array}{l}722 \\
576\end{array}$ & 2 & $\begin{array}{l}242 \\
137\end{array}$ & $\begin{array}{l}458 \\
774\end{array}$ & $\begin{array}{l}13.5 \\
11.2\end{array}$ & & $\begin{array}{l}942 \\
112\end{array}$ & $\begin{array}{l}102 \\
670\end{array}$ & $\begin{array}{l}42.0 \\
81.8\end{array}$ \\
\hline \multicolumn{13}{|l|}{1940} \\
\hline $\begin{array}{l}\text { México } \\
\text { Distrito }\end{array}$ & Federal & $\begin{array}{r}19 \\
1\end{array}$ & $\begin{array}{l}653 \\
757\end{array}$ & $\begin{array}{l}552 \\
530\end{array}$ & 4 & $\begin{array}{l}323 \\
342\end{array}$ & $\begin{array}{l}781 \\
718\end{array}$ & $\begin{array}{l}22.0 \\
19.5\end{array}$ & 1 & $\begin{array}{l}960 \\
228\end{array}$ & $\begin{array}{l}755 \\
413\end{array}$ & $\begin{array}{l}45.3 \\
66.6\end{array}$ \\
\hline \multicolumn{13}{|l|}{1950} \\
\hline $\begin{array}{l}\text { México } \\
\text { Distrito }\end{array}$ & Federal & $\begin{array}{r}25 \\
3\end{array}$ & $\begin{array}{l}791 \\
050\end{array}$ & $\begin{array}{l}017 \\
442\end{array}$ & 6 & $\begin{array}{l}074 \\
616\end{array}$ & $\begin{array}{l}486 \\
631\end{array}$ & $\begin{array}{l}23.6 \\
20.2\end{array}$ & 4 & $\begin{array}{l}026 \\
432\end{array}$ & $\begin{array}{l}691 \\
268\end{array}$ & $\begin{array}{l}49.8 \\
70.1\end{array}$ \\
\hline \multicolumn{13}{|l|}{1960} \\
\hline $\begin{array}{l}\text { Mêxico } \\
\text { Distrito }\end{array}$ & Federal & $\begin{array}{r}34 \\
4\end{array}$ & $\begin{array}{l}923 \\
870\end{array}$ & $\begin{array}{l}129 \\
876\end{array}$ & $\begin{array}{l}8 \\
1\end{array}$ & $\begin{array}{l}516 \\
064\end{array}$ & $\begin{array}{l}316 \\
801\end{array}$ & $\begin{array}{l}24.4 \\
21.9\end{array}$ & 5 & $\begin{array}{l}401 \\
903\end{array}$ & $\begin{array}{l}509 \\
097\end{array}$ & $\begin{array}{l}63.4 \\
84.8\end{array}$ \\
\hline 1970 & & & & & & & & . & & & & \\
\hline $\begin{array}{l}\text { México } \\
\text { Distrito }\end{array}$ & Federai & $\begin{array}{r}48 \\
6\end{array}$ & $\begin{array}{l}225 \\
874\end{array}$ & $\begin{array}{l}238 \\
16\end{array}$ & $\begin{array}{c}12 \\
1\end{array}$ & $\begin{array}{l}431 \\
590\end{array}$ & $\begin{array}{l}880 \\
097\end{array}$ & $\begin{array}{l}25.8 \\
23.1\end{array}$ & $\begin{array}{l}7 \\
1\end{array}$ & $\begin{array}{l}358 \\
081\end{array}$ & $\begin{array}{l}017 \\
385\end{array}$ & $\begin{array}{l}59.2 \\
68.0\end{array}$ \\
\hline
\end{tabular}

FUENTE: Cuadro elaborado a partir de los cuadros 2 y 4 (sección Educación); 53 y 6.6 (Educación y cultura) del Anuario Estadístico de los Estados Unidos Mexi. canos para los años de 1930, 1950 y 1960 y de los cuadros 19; Primaria; 3-A; 8 y 3 del Censo General de la Población, Resumen General, para los años 1930, $1940,1950,1960$ y 1970.

a Para el año 1930 se refiere a la población de 6 a 10 años de edad. 
El cuadro 2 muestra la demanda potencial ${ }^{2}$ y la matrícula escolar primaria. Dos hechos son los más sobresalientes al respecto:

a) A pesar de que la demanda potencial ha ido en aumento a un ritmo relativamente lento tanto en el país como en el Distrito Federal (existe una anomalía para el año 1930 debida al cambio en los límites de edad), su aumento no ha sido acorde con el crecimiento de la población, pues si así fuese, los valores relativos de la demanda potencial, entre un año y otro serían superiores a los observados.

b) La matrícula escolar primaria, tanto en término absolutos como relativos, ha ido en aumento. En el caso del país el sistema educativo no ha podido en ningún momento acoger ni siquiera al $75 \%$ de la demanda potencial primaria, lo que demuestra cierta pobreza en su capacidad de absorción. No obstante, en el Distrito Federal, a pesar de que la información es fluctuante, lo que impide llegar a conclusiones precisas sobre la tendencia general, la matrícula escolar es, en todos los años considerados, superior a la observada para el total del país.

El cuadro 3, además de permitir observar diferencias rural-urbanas, al

\section{Cuadro 3}

MOVIMIENTO Y APROVECHAMIENTO DE LOS ALUMNOS EN LAS ESCUELAS primarias uRbanas Y RURALES EN México Y EL Distrito Federal, 1950,1960 y 1970

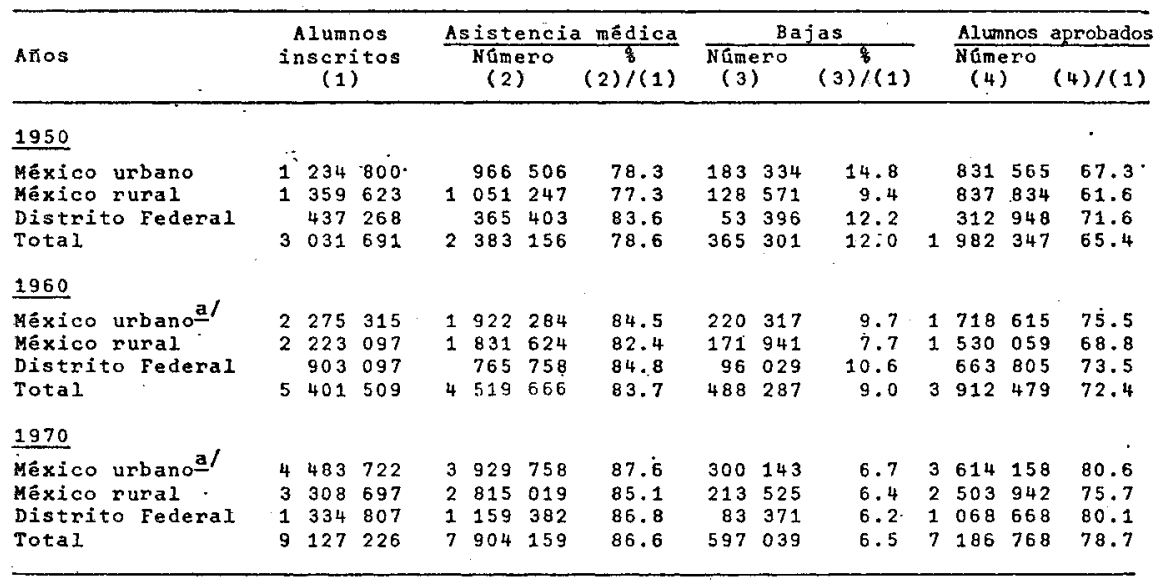

FueNTE: Elaborado a partir de los cuadros 59, 60, 64 y 65; 6.40, 6.42, 6.44 y $6.46 ; 6.15,6.17,6.19$ y 6.21 del Anuario estadistico de los Estados Unidos Me. xicanos Años 1950, 1960 y 1970.

a No incluye al Distrito Federal.

2 Se entiende por demanda potencial la población que se encuentra dentro de los grupos de edades que, en un sistema escolar plenamente normalizado, estarían comprendidos por los distintos niveles escolares. En este caso, la demanda potencial de enseñanza primaria está formada por los niños que tienen entre 6 y 14 años. 
mostrar el movimiento y aprovechamiento de los alumnos en las escuelas primarias, brinda la oportunidad de apreciar en parte la eficiencia interna del sistema educativo.

A pesar de que el citado cuadro sólo pudo ser elaborado para los años 1950,1960 y 1970 , permite apreciar que el Distrito Federal presenta una posición bastante satisfactoria, si se compara con el medio urbano y, mejor aún, si la observación se hace en relación al medio rural. Puede decirse que, para casi todos los años considerados, registra la proporción más elevada de asistencia media, la menor en cuanto a bajas (al menos en el último año) y una buena proporción de alumnos aprobados.

De manera similar al caso anterior, el cuadro 4, que muestra la existencia de las escuelas primarias según el número de grados que se imparten, complementa la idea sobre la expansión del sistema educativo.

El Distrito Federal, al igual que en las situaciones examinadas antes, también presenta en este caso una situación de ventaja, ya que para 1960 , último año para el que se dispone de información, el $87 \%$ de las escuelas primarias contaban con los seis grados de enseñanza. Esta situación está muy lejos de ser igualada en el medio rural, en donde para 1970 sólo el $19.6 \%$ de las escuelas tenían el ciclo de educación primaria completa.

\section{Cuadro 4}

Escuelas primarias uRbaNas Y RURALES, POR GRADOS, EN MÉxico Y EL Distrito Federal, 1950, 1960 y 1970

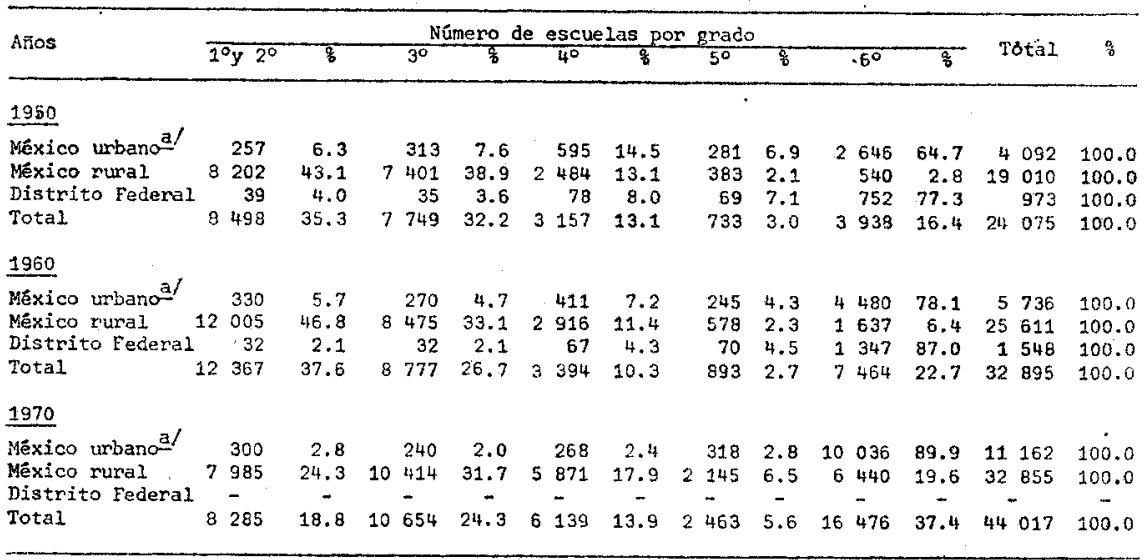

FuENTE: Elaborado a partir de los cuadros 57, 6.21 y 6.9 del Anuario Estadistico de los Estados Unidos Mexicanos, 1950, 1960 y 1970.

a No incluye al Distrito Federal.

El análisi del cuadro 5, también ofrece la oportunidad de apreciar diferencias rural-urbanas y, de observar aún en mejor forma la expansión del sistema educativo. 
En este caso, más que las cifras absolutas, conviene observar los índices que se pueden obtener con dichas cifras. Así, por ejemplo, la proporción de alumnos por escuela ha estado aumentando de manera constante desde 1927. Dicha presión afecta más al sector urbano y al Distrito Federal, lo cual es consecuencia del crecimiento de la población urbana, producto en buena parte de la emigración rural-urbana. Este incremento cada vez mayor del número de alumnos por escuela da una idea de la presión que debe enfrentar y resolver el sistema educativo mexicano.

En relación a la proporción de alumnos por maestro, no se pueden hacer mayores elucubraciones, dada la manera como fluctúa dicha relación. Sin embargo, todo parece indicar que es en el Distrito Federal donde se observa que dicha proporción ha ido en aumento, pero se piensa que ello no constituye una situación crítica, dado que es también en el Distrito Fe-

\section{Cuadro 5}

NÚMERO DE ESCUELAS, ALUMNOS INSCRITOS, ALUMNOS POR ESCUELA, PERSONAL DOCENTE, ALUMNOS POR MAESTRO Y MAESTROS POR ESCUELA, EN MÉXICo Y EL Distrito FEDERAL, 1927, 1940, 1950, 1960 y 1970

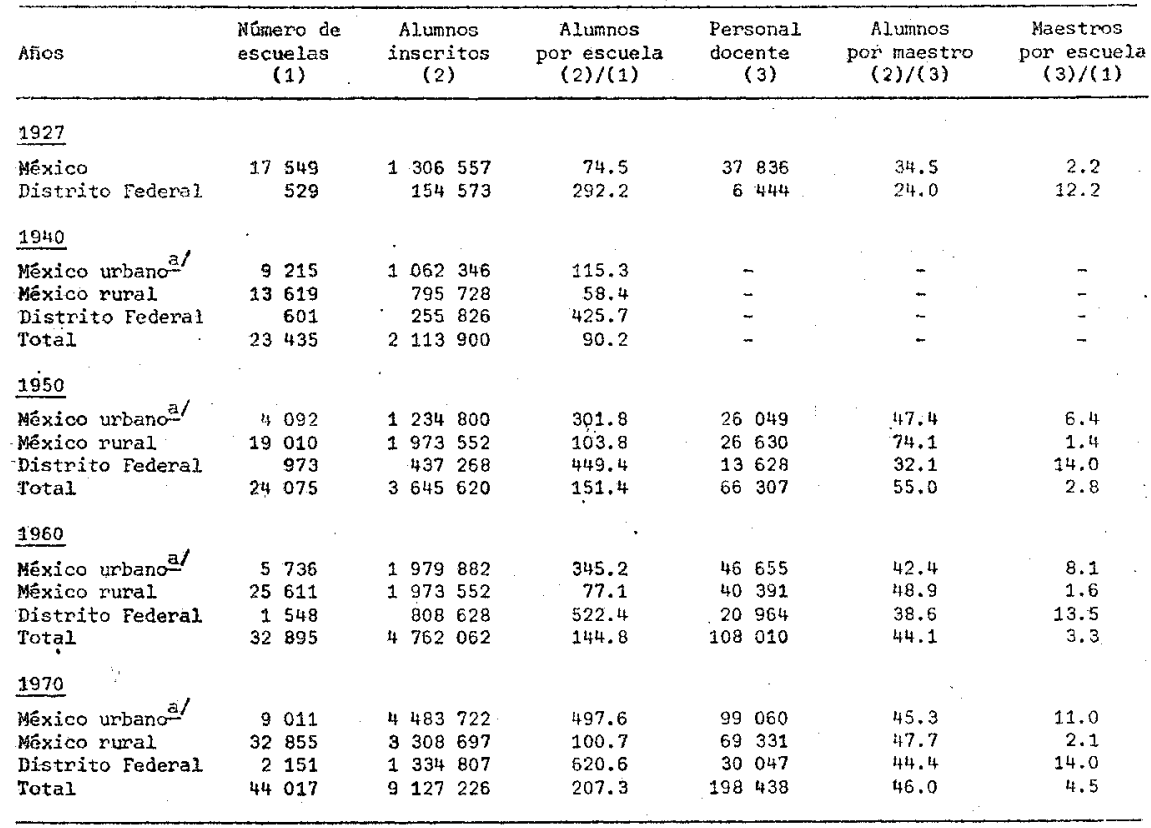

FUENTE: Elaborado a partir de los cuadros 1,2 y $3 ; 180$ y $182 ; 57$ y $66 ; 6.28$, 6.31 y 6.10 del Anuario Estadístico de los Estados Unidos Mexicanos, para los años 1930, 1942, 1950, 1960 y 1970.

a No incluye al Distrito Federal. 
deral donde se aprecia una mayor proporción de maestros por escuela, lo que hasta cierto punto puede compensar la primera situación.

La última proporción analizada (maestros por escuela), no muestra otra cosa que la desventaja tan grande en que se encuentra el medio rural, en donde dicha proporción, además de haber permanecido prácticamente inmóvil, revela para el año 1970 el ínfimo valor de 2.1 maestros por escuela.

Al igual que en las situaciones examinadas en los cuadros que preceden, el Distrito Federal en este caso también muestra una posición de ventaja, ya que a través de todo el tiempo considerado registra la menor proporción de alumnos por maestro y la mayor proporción de maestros por escuela.

La caracterización del sistema educativo, además de lo expresado en la visión global, se podría complementar a través del análisis de diferencias regionales y de diferencias a nivel de estratos sociales.

En el primero de los casos, dice Muñoz Izquierdo (3, 1973, p. 32) que al utilizar la clasificación que según la riqueza realizó Wilkie en 1970, se observa que tanto en 1960 como en 1970 existía una clara asociación entre la riqueza económica y la riqueza educativa en las siete regiones. que comprendió la regionalización, es decir, los recursos dedicados a la educación son aprovechados, en primer lugar, por las entidades que desde otros puntos de vista han sido más favorecidas y, sólo hasta el momento en que dichas entidades han alcanzado niveles de desarrollo escolar lo suficiente altos, los recursos empiezan a fluir hacia las regiones más rezagadas.

En cuanto a las diferencias a nivel de estratos sociales, ha sido poco lo que se ha investigado, o al menos lo que se ha publicado al respecto in México, Muñoz Izquierdo, en su trabajo sobre la Evaluación del desarrollo educativo en México (1958-1970) y factores que lo han determinado, señala que sólo una investigación de alcance nacional y referida en exclusiva a la enseñanza primaria se ha realizado. Su finalidad fue detectar la relación existente entre la posición social de los individuos y la dosis de escolaridad a que tienen acceso. El estudio reveló que de 7887869 niños de 6 a 14 años de edad, 3057596 se encontraban fuera de la escuela primaria y que a su vez 1400000 niños estaban imposibilitados para asistir a la escuela, por razones de carácter económico.

Indica además que: "el $40 \%$ de esos niños pertenecía a familias cuyos ingresos mensuales eran inferiores a 200 pesos, el $30.6 \%$ a familias con ingresos entre 201 y 400 pesos, el $13.2 \%$ a familias con ingresos entre 401 y 600 pesos, y el $9.8 \%$ a familias con ingresos entre 601 y 1000 pesos mensuales"' (p. 25). Como se desprende de lo expuesto, el estudio mostró una correlación inversa entre los estratos de ingreso familiar y la proporción de los componentes de cada estrato que no pueden asistir a la escuela por las razones indicadas. También comprobó que la perseveración en el 
sistema escolar, hasta concluir la educación primaria, está asociada con los niveles de ingresos de las familias a que pertenecen los alumnos.

Estudios realizados por el Centro de Investigaciones Económicas de la Facultad de Economía de la Universidad de Nuevo León, y el Centro de Investigaciones sobre Población de la Universidad de Texas en el año $1967,{ }^{3}$ con la finalidad de establecer la relación entre el acceso a la enseñanza media y superior y la posición social de las personas, revelaron que tanto el estrato social, como la escolaridad alcanzada por los padres de los individuos, son importantes en la determinación de la distribución de oportunidades escolares en todos los niveles de enseñanza.

Latapí $(4,1973)$, en su libro Mitos y verdades de la educación mexicana, 1971-1972, con la finalidad de mostrar que el mito más acariciado de la política educativa en México, expresión que tomamos del autor en su forma original, es el de adjudicarle a la educación un efecto mágico sobre la movilidad social, hace un análisis de la manera como la enseñanza ha incidido en las diferentes clases sociales. Se anotan a continuación los resultados en forma resumida.

La clase baja mexicana recibe oportunidades educativas que no están en proporción con las prédicas políticas de igualdad de que tanto se habla, pues los grupos indígenas permanecen prácticamente inmóviles en su condición de marginados y los campesinos no cuentan con escuelas primarias completas; la eficiencia terminal es muy baja; la oferta de enseñanza secundaria es muy reducida y; las oportunidades post-primarias de adiestramiento para el trabajo, se puede decir que no existen.

El sector obrero, casi siempre sujeto, como lo expresa Latapí, a un régimen de beneficios a cambio de sumisión, nunca se ha distinguido por presiones tendientes a ampliar sus oportunidades de educación.

La clase media, de manera principal sus estratos superiores, es quien más se ha aprovechado de la educación para lograr cierto ascenso social, razón por la cual puede afirmarse que la educación nacional, más que populista es elitista, en la medida en que favorece la transición ascendente dentro de la propia clase media $\mathrm{y}$, en menor proporción, de ésta hacia la clase alta.

La clase alta (grupos privilegiados económica y políticamente), no es en México, según la opinión de Latapí, una clase de elevada educación y, por lo tanto, carece de una fuerte tradición cultural. Sus miembros, una vez establecidos, se aislan de las otras clases y olvidan los mecanismos por medio de los cuales llegaron allí. No obstante, tienen muchas maneras de mantener su poder, de manera principal concediendo beneficios a quienes no los pueden obtener por otros medios. No ven en la educación la fuente principal de su ascenso y permanencia en la cúspide social, pero sí se preo-

3 Para mayor información a este respecto, también pueden verse los trabajos de: Jorge Balán, The Process of Stratification in an Industrializing Society. Tesis doctoral, Universidad de Texas y; Jesús Puente Leyva, La distribución del ingreso en un área metropolitana: El caso de Monterrey, México, Siglo XXI. 
cupan porque la educación transmita los valores propios de su clase, para perpetuar el sistema de ascenso del que se han beneficiado.

Este breve resumen del comportamiento de las clases sociales en relación con la educación, muestra lo discutible que es afirmar que en México existe una relación armoniosa entre la educación y la justicia social.

De todo lo expuesto se podría concluir que no sería justo negar la considerable expansión de los servicios educativos, incluso en las zonas rurales pero que, como bien lo expresa Medellín (5, 1973, pp. 102-103), al ser el sistema educativo (su evolución y funcionamiento), por una parte, el resultado de la interacción de diversos factores, entre los cuales el esquema de desarrollo económico y el sistema de estratificación social juegan un papel muy importante; y, por la otra, al ser también el sistema educativo un reflejo de la estructura de poder y uno de los mecanismos mediante los cuales se reproduce y mantiene dicha estructura, él mismo ha operado de una manera inorgánica e ineficaz, de tal modo que aún subsisten muchos problemas que ha sido incapaz de resolver.

Es por eso que el sistema escolar sigue mostrando una configuración marcadamente piramidal, en donde se aprecia que la eficiencia interna del mismo no se ha traducido en garantizar oportunidades sociales uniformes del tipo que se ofrezcan, sino en seguir favoreciendo de manera principal a las comunidades urbanas, a las regiones sociogeográficas de mayor desarrollo, al Distrito Federal y a los sectores sociales altos.

\section{Hipótesis de trabajo}

La hipótesis de trabajo se desprende de las observaciones encontradas en la caracterización de los rasgos más sobresalientes de la situación educativa en el país y en la ciudad de México, y de las deducciones a que condujo la revisión bibliográfica de los trabajos que en América Latina han analizado el diferencial fecundidad-escolaridad."

Se parte de la existencia de una asociación entre el nivel de fecundidad y el grado de escolaridad de las mujeres, con la salvedad de que dicha

4 La revisión bibliográfica a que se hace mención la hizo Zambrano Lupi (7, 1977), en La relación entre la fecundidad y el grado de escolaridad en el medio rural mexicano y en la ciudad de México. Alli se analizaron los trabajos de Virginia Rodríguez (1968), M. J. Elsa Cerisola (1968), Alfredo Enrique Lates (1967), Severo Rivera (sin fecha), J. Mayone Stycos (1970), Walter Mertens (1970), Robert Carleton (1965-1970), Zulma Camisa (1976) y Catalina Gougain (1977). La mayoría de dichos trabajos se orientan bajo el enfoque de la fecundidad diferencial, a excepción de los de Carleton, dada su naturaleza principalmente térica. Asimismo, al margen de que contengan una formulación previa al problema, acompañado o no de la postulación de hipótesis y de que el análisis de la relación fecundidad-escolaridad se haya hecho de manera directa o mediante técnicas de tipificación, las investigaciones cubiertas evidenciaron, con mayor o menor fundamento, la existencia de una asociación negativa entre el nivel de fecundidad y el grado de escolaridad. 
asociación, aunque por lo general lo sea, no siempre es negativa y uniforme.

La postulación de dicha asociación obedece a evidencias empíricas observadas en los variados resultados encontrados sobre el comportamiento de la relación entre la fecundidad y el grado de escolaridad en los estudios examinados en la revisión bibliográfica. A ello se agregan los múltiples hallazgos que sobre el comportamiento de la misma relación describe Serim Timur $(6,1977)$, en su trabajo Variables demográficas correlacionadas con la edad de la mujer: Fecundidad, edad al matrimonio y familia, en más de treinta países, en su mayoría asiáticos y africanos, catalogados en tres grupos conforme a la posición de los mismos en cuanto a la transición demográfica.

Por otra parte, se afirma que la relación no tiene que ser por necesidad siempre negativa y uniforme por dos razones. En primer lugar, recordemos que se trata de un vínculo ineludiblemente cambiable, que revela la variabilidad de los comportamientos humanos con las circunstancias sociales. En segundo lugar, aunque las dos variables (fecundidad y escolaridad), estén asociadas, no se puede esperar que siempre se afecten por igual: el grado de asociación variará en función de los factores involucrados con cada una de ellas, lo que le resta validez al contenido "necesariamente uniforme" por lo común se le asigna a dicha asociación.

Formuladas estas breves premisas teóricas se establece nuestra hipótesis de trabajo: En la ciudad de México, de manera más factible que en el medio rural, la asociación entre el nivel de fecundidad y el grado de escolaridad, es de esperar que se comporte como una relación negativa y uniforme. Dicha relación, una vez que se introduzca alguna referencia a la posición social de los individuos y que se progrese en la escala de la estratificación, es razonable que adquiera mayor consistencia y uniformidad.

Esta hipótesis destaca a la urbanización como portadora de factores que inciden en la reducción de la fecundidad. A continuación se tratará de elucidar de dónde se derivan esos factores y de justificar la formulación de la hipótesis:

1) Se debe tener presente que -incluso antes de engendrarse el proceso de urbanización, tal como se le conoce hoy día- la desigualdad ciudad-campo, en términos de nivel social, económico y cultural, siempre ha existido bajo una relación que, como se expresó antes, por lo regular ha favorecido a la ciudad.

2) Bajo condiciones de capitalismo dependiente, como es el caso de México, lo primero que hay que tener en cuenta es que las relaciones de dominación entre ciudad y campo, como lo expresa Paul Singer (8, 1975, pp. 8-10), si no se hace abstracción de las relaciones de clase, lo que expresan son los vínculos de dominación de clases entre el medio urbano y el rural. La ciudad es, en general, la sede del poder y por lo tanto de la clase dominante, y el campo, el sector en donde se produce la sobre- 
ganancia agrícola, que el capitalismo industrial intercepta mediante todo un dispositivo y canaliza hacia afuera del sector agrícola, de tal modo que la evolución de dicho sector queda regulada de manera directa por el desarrollo del capitalismo industrial.

3) La relación de dependencia campo-ciudad expresada en el párrafo anterior, conforme a la concepción de Aníbal Quijano (9, 1975, p. 42), no debe ser vista en función de un dualismo estructural, que divida lo urbano y lo rural en dos estructuras sociales de naturaleza histórica diferente. Así lo ha concebido la famosa "teoría de la modernización" al hablar de los polos tradicional y moderno.

Dicha relación hay que entenderla como un fenómeno en donde lo urbano y la rural forman parte de una misma evolución global. En ella los procesos y las tendencias de cambio que se dan en cada nivel son correspondientes, y ambos en su conjunto constituyen el proceso global de cambio, aunque la acción recíproca no se verifique con la misma intensidad entre ellas sino, como se expresó antes, bajo una posición de primacía de la ciudad en relación al campo.

4) Dentro del proceso de urbanización dependiente que ha venido caracterizando a la mayoría de los países latinoamericanos que giran bajo la órbita del capitalismo contemporáneo, de los cuales México no es una excepción, dicha urbanización ha favorecido a pocas áreas y ciudades.

5) Ese comportamiento que ha caracterizado a la urbanización dependiente, según la expresión de Marcos Kaplan (10, 1973, p. 140), no es otra cosa que la selección de que han sido objeto aquellas ciudades y regiones adecuadas y privilegiadas, y que por lo regular son ejes de acceso al país por parte de los grupos que controlan el comercio exterior y las inversiones extranjeras.

6) Tales ciudades, al funcionar y operar como bases, polos, ejes, etcétera, de las estructuras, instituciones y mecanismos que se identifican o vinculan con el movimiento del comercio internacional y de las inversiones extranjeras, como bien lo apunta Kaplan (pp. 138-139), no sólo son las sedes de las instituciones políticas y del Estado. Se constituyen cada vez más en centros de poder, en intermediarias dependientes entre las metrópolis externas y sus corporaciones internacionales, por una parte, y el resto del país, en especial el hinterland rural, por otra.

7) De lo expuesto antes, no es entonces una casualidad, ni tampoco debe extrañar que el análisis del sistema educativo mexicano muestre que quien mejor se ha aprovechado de su expansión, en todos los sentidos, ha sido el Distrito Federal, sede de la ciudad capital del país.

8) Del párrafo anterior se desprende que la situación educacional de las mujeres en la ciudad de México ocupa un lugar de primacía en relación al país: menor analfabetismo; mayor número de escuelas con primaria completa; mayor número de maestros; el movimiento y aprovechamiento de los alumnos presenta características muy positivas; la educación 
no sólo se limita a la primaria, sino que se complementa con la secundaria superior y de postgrado, etc.

9) En el medio urbano y a medida que la proporción de escolaridad recibida es mayor y se asciende en la escala social del trabajo, es más lógico pensar en el papel que puede jugar la escolaridad como agente de movilidad social expresada, entre otras cosas, en una mejor perspectiva de vida. Esta exige modificaciones en todo sentido, estando entre los efectos de esas modificaciones los cambios en el comportamiento reproductivo.

10) En las ciudades, aunque no siempre, pero sí en muchos casos, el contenido de la escolaridad recibida va acompañado de otros factores que complementan la acción de dicha escolaridad. Entre otros se pueden citar los siguientes: la emancipación de la mujer es más factible; las oportunidades de trabajo de la mujer son mayores; todo parece indicar que cobra más importancia la responsabilidad de los padres, en el sentido de asegurarle a sus hijos una educación superior a la que ellos recibieron, no sólo en cantidad sino en calidad, etc. A ello se puede agregar que en el medio urbano es más factible que la escolaridad per capita, si no va acompañada de una mejor distribución de la riqueza, al menos resulte que el aumento en el número de empleos esté en mayor correspondencia con la cantidad de personas instruidas.

Por otra parte, la organización social del trabajo, más desarrollada en el medio urbano que en el rural, exige a los habitantes de la ciudad una mayor dosis de escolaridad para el mejor desempeño de las labores urbanas.

Expuestas algunas razones que justifican la formulación de nuestra hipótesis, queda explícito que la relación entre la fecundidad y el grado de escolaridad que se espera encontrar en la ciudad de México, no puede ser otra que la propia del contexto socioeconómico donde se lleva a cabo dicha relación. Es decir, ni siquiera por tratarse de un medio urbano como lo es la ciudad de México se deben esperar resultados similares a los observados en economías más desarrolladas, porque sería ilógico suponer comportamientos ante la reproducción similares a la de aquellos países, en medios donde el proceso es de naturaleza diferente.

\section{ANÁlISIS DE LA RELACIÓN ENTRE FECUNDIDAD Y GRADO DE ESCOLARIDAD EN LA CIUdAD DE MÉxico ${ }^{5}$}

1. Distribución de las mujeres entrevistadas casadas y convivientes, según la edad y el grado de escolaridad

En relación a la distribución por edad de las mujeres casadas y convivientes puede decirse que la proporción de mujeres entrevistadas es muy

5 El nivel de la fecundidad estará dado por el número medio de hijos nacidos vivos de las mujeres casadas y convivientes cubiertas por la encuesta. Por su parte, el grado de escolaridad se corresponde con el conjunto de los cursos alcanzados por las mujeres casadas y convivientes. 
semejante entre las edades 20 y 39 años, y que sólo los grupos 15-19, y 40 años y más, no presentan esa característica (véase el cuadro 6).

\section{Cuadro 6}

Ciudad de México: Distribución de las mujeres entrevistadas, CASADAS Y CONVIVIENTES POR GRUPOS DE EDAD

\begin{tabular}{rrr}
\hline Grupos de edad & Entrevistadas & \% \\
\hline $15-19$ & 51 & 3.5 \\
$20-24$ & 232 & 16.0 \\
$25-29$ & 284 & 19.5 \\
$30-34$ & 286 & 19.7 \\
$35-39$ & 253 & 17.4 \\
$40-44$ & 186 & 12.8 \\
$45-49$ & 161 & 11.1 \\
Total & 1453 & 100.0 \\
\hline
\end{tabular}

Fuente: Fase A de la Encuesta de Migración a la ciudad de México, 1970.

La proporción tan reducida de mujeres casadas y convivientes, en el grupo 15-19 años, podría hacer dudar de la confiabilidad de la muestra. No obstante, Goldani $(11,1977$, p. 43), encontró que, en términos generales, la distribución porcentual por edades entre la población del área metropolitana en el censo y en la encuesta no difieren mayormente. Aunque la comparación realizada por Goldani es de carácter más general (compara la población total y no la población casada y conviviente), el haber comprobado la distribución total, hace pensar que la proporción de las mujeres casadas y convivientes, debe también responder a su repartición dentro de la población censal.

En virtud de que para la realización en este apartado se trabajó con base en la información de la muestra de la Fase $A$, de la encuesta sobre Migración interna, estructura ocupacional y movilidad social en el área metropolitana de la ciudad de México, la ciudad de México, para los efectos de este trabajo, comprende el área que cubrió la mencionada muestra, la cual difiere de los límites políticos del Distrito Federal. Se eliminaron algunas regiones que por estar privadas de los servicios públicos más elementales y, por caracterizarse por una baja densidad demográfica y una economía principalmente agropecuaria, se identificaron más como zonas rurales que urbanas.

De manera precisa la ciudad de México quedó definida por el Distrito Federal menos las Delegaciones de Cuajimalpa, Tláhuac y Milpa Alta, las cuales fueron eliminadas en su totalidad. También se eliminaron las partes rurales de las Delegaciones de Obregón, Magdalena Contreras, Tlalpan y Xochimilco, considerándose de estas Delegaciones sólo las partes urbanas más importantes aledañas a las zonas densamente pobladas del Distrito Federal. A su vez, se incluyeron como parte de la ciudad de México, los municipios de Naucalpan, Tlalnepantla, Ecatepec, Netzahualcóyotl y Chimalhuacán, los cuales, a pesar de estar ubicados en el Estado de México se integraron como parte de la ciudad por el hecho de conservar con el Distrito Federal una continuidad urbana en términos de servicios, comunicaciones y tipo de actividad económica. 
La distribución de las mujeres casadas y convivientes según su grado de escolaridad, viene desplegada originalmente en la encuesta en ocho categorías, incluida la "No sabe". Como puede apreciarse en el cuadro 7 , el $14.4 \%$ de las mujeres entrevistadas no había realizado ninguna clase de estudios, el 51.7\%, proporción bastante significativa, correspondió a las mujeres con algún grado de educación primaria, aunque sólo el $18.8 \%$ alcanzó la primaria completa, mientras que las mujeres con una educación superior a la primaria completa constituyen el $33.6 \%$. Este último hecho es bastante significativo, si recordamos que el medio rural mexicano dicha proporción sólo alcanzaba $3.5 \%$ en $1970 .^{6}$ Ello no debe extrañar gran cosa si se tiene en cuenta que la expansión del sistema educativo en México se ha venido haciendo en todos los sentidos, en beneficio del medio urbano, y de manera principal del Distrito Federal.

\section{Cuadro 7}

Ciudad de México: Distribución de las mujeres entrevistadas, CASADAS Y CONVIVIENTES SEGÚN GRADO DE ESCOLARIDAD

\begin{tabular}{lcr}
\hline Grados de escolaridad & Entrevistadas & \% \\
\hline Sin educación & 209 & 14.4 \\
10 a 30 $^{\circ}$ a 50 & 293 & 20.2 \\
Primaria completa & 185 & 12.7 \\
Secundaria & 273 & 18.8 \\
Preparataria & 349 & 24.0 \\
Universidad & 91 & 3.3 \\
Ho sabe & 48 & 0.3 \\
Total & 5 & 100.0 \\
\hline
\end{tabular}

Fugnte: Fase A de la Encuesta de Migración a la ciudad de México, 1970.

La distribución de las mujeres entrevistadas casadas y convivientes, según la edad y el grado de escolaridad, una vez hecha la agrupación de esta última variable en cinco niveles, aparece en el cuadro 8.

Dos hechos resaltan al observar dicho cuadro. El primero es que si bien es cierto que la mayor proporción de mujeres entrevistadas se ubican en el nivel de 0-3 grados de escolaridad, a medida que dicha escolaridad aumenta, la proporción de mujeres varía, pero no necesariamente disminuye. Es más, en casi todos los grupos de edades aumenta la proporción para la primaria completa y para la secundaria, en relación al porciento de las que sólo llegaron al nivel de 4-5. Ello no debe extrañar si se recuerda que en el Distrito Federal, para el año 1960 ya el $87 \%$ de las escuelas contaban con los seis grados de primaria, además de que existen numerosos centros de educación secundaria, situación que no se da en el medio rural.

El otro hecho significativo es que no sólo las mujeres más jóvenes (entre 15 y 34 años), alcanzan los niveles más elevados de escolaridad. Tam-

6 Datos obtenidos de la encuesta de fecundidad rural de 1970. 
bién la proporción de mujeres de 35 años y más es significativa en este sentido en el Distrito Federal.

\section{Cuadro 8}

Ciudad de MÉxico: Distribución de las mujeres entrevistadas, casadas Y CONVIVIENTES, SEGÚN GRUPOS QUINQUENALES DE EDAD Y NIVEL DE ESCOLARIDAD

\begin{tabular}{|c|c|c|c|c|c|c|c|c|}
\hline \multirow[b]{2}{*}{ Grupos de } & \multirow[b]{2}{*}{ edad } & \multicolumn{6}{|c|}{ Grado de escolaridad } & \multirow[b]{2}{*}{ Total } \\
\hline & & $0-3$ & $4-5$ & $\begin{array}{l}\text { Primaria } \\
\text { completa }\end{array}$ & Secundaria & $\begin{array}{l}\text { Preparatoria } \\
\text { y universidad }\end{array}$ & no sabe & \\
\hline \multicolumn{9}{|l|}{$15-19$} \\
\hline Mujeres & & $\begin{array}{c}18 \\
35.3\end{array}$ & $\begin{array}{c}10 \\
19.6\end{array}$ & $\begin{array}{r}15 \\
29.4\end{array}$ & 13.7 & 2.00 & 0.00 & $\begin{array}{c}51 \\
100.0\end{array}$ \\
\hline \multicolumn{9}{|l|}{$20-24$} \\
\hline Mujeres & & $\begin{array}{c}63 \\
27.2\end{array}$ & $\begin{array}{c}35 \\
15.1\end{array}$ & $\begin{array}{c}45 \\
19.4\end{array}$ & $\begin{array}{c}66 \\
28.4\end{array}$ & $\begin{array}{r}23 \\
9.9\end{array}$ & $\begin{array}{l}0 \\
0.00\end{array}$ & $\begin{array}{c}232 \\
100.0\end{array}$ \\
\hline \multicolumn{9}{|l|}{$25-29$} \\
\hline $\begin{array}{l}\text { Mujeres } \\
\%\end{array}$ & & $\begin{array}{c}83 \\
29.2\end{array}$ & $\begin{array}{l}27 \\
9.5\end{array}$ & $\begin{array}{l}54 \\
19.0\end{array}$ & $\begin{array}{c}84 \\
29.6\end{array}$ & $\begin{array}{r}35 \\
12.3\end{array}$ & $\begin{array}{c}1 \\
0.4\end{array}$ & $\begin{array}{l}284 \\
\$ 00.0\end{array}$ \\
\hline $30-34$ & & . & & & & & & \\
\hline $\begin{array}{l}\text { Mujeres } \\
\$\end{array}$ & & $\begin{array}{l}108 \\
37.8\end{array}$ & $\begin{array}{c}31 \\
10.8\end{array}$ & $\begin{array}{l}50 \\
17.5\end{array}$ & $\begin{array}{l}53 \\
22.0\end{array}$ & $\begin{array}{r}33 \\
11.5\end{array}$ & $\stackrel{1}{0.4}$ & $\begin{array}{l}286 \\
100.0\end{array}$ \\
\hline \multicolumn{9}{|l|}{$35-39$} \\
\hline$\underset{z}{M}$ ujeres & & $\begin{array}{c}89 \\
35.2\end{array}$ & $\begin{array}{c}40 \\
15.8\end{array}$ & $\begin{array}{c}41 \\
16.2\end{array}$ & $\begin{array}{c}63 \\
24.9\end{array}$ & $\begin{array}{r}19 \\
7.5\end{array}$ & $\begin{array}{c}1 \\
0.4\end{array}$ & $\begin{array}{c}253 \\
100.0\end{array}$ \\
\hline \multicolumn{9}{|l|}{$40-44$} \\
\hline Mujeres & & $\begin{array}{c}74 \\
39.8\end{array}$ & $\begin{array}{c}20 \\
10.8\end{array}$ & $\begin{array}{c}35 \\
18.8\end{array}$ & $\begin{array}{c}41 \\
22.0\end{array}$ & $\begin{array}{r}15 \\
8.1\end{array}$ & ${ }^{1}$ & $\begin{array}{c}186 \\
100.0\end{array}$ \\
\hline \multicolumn{9}{|l|}{$45-49$} \\
\hline Mujeres & & $\begin{array}{l}67 \\
41.6\end{array}$ & $\begin{array}{l}22 \\
13.7\end{array}$ & $\begin{array}{c}33 \\
20.5\end{array}$ & $\begin{array}{l}25 \\
15.5\end{array}$ & $\begin{array}{r}13 \\
8.1\end{array}$ & $\begin{array}{c}1 \\
a .6\end{array}$ & $\begin{array}{l}161 \\
100.0\end{array}$ \\
\hline \multicolumn{9}{|l|}{ Tota1 } \\
\hline Mujeres & & $\begin{array}{l}502 \\
34.5\end{array}$ & $\begin{array}{l}185 \\
12.7\end{array}$ & $\begin{array}{l}273 \\
18.8\end{array}$ & $\begin{array}{l}349 \\
24.0\end{array}$ & $\begin{array}{l}139 \\
9.6\end{array}$ & 5.4 & $\begin{array}{l}1453 \\
100.0\end{array}$ \\
\hline
\end{tabular}

Fuente: Fase A de la Encuesta de Migración a la ciudad de México, 1970.

2. Análisis de la relación entre la fecundidad y el grado de escolaridad, según grupos quinquenales de edad de las madres

La influencia que pueda ejercer el grado de escolaridad en la fecundidad será analizada manteniendo constante la edad de las madres en el momento de la encuesta. Se sabe que este medio de control, al no tener en cuenta la duración de la unión, no mide de manera rigurosa la exposición al riesgo de tener hijos a que han estado expuestas las mujeres. Constituye más bien una aproximación en ese sentido. 
En el cuadro 9, aparece la evolución del número medio de hijos nacidos vivos bajo las condiciones mencionadas antes.?

El primer aspecto notable es que el número medio de hijos nacidos vivos de las mujeres casadas y convivientes de la ciudad de México es de 4.20, cifra que en términos medios muestra una diferencia de 1.09 hijos menos, en relación al medio rural mexicano (véase, Zambrano, Lupi, 7 , 1977). El número medio de hijos nacidos vivos disminuye a medida que el nivel de escolaridad aumenta, de manera similar al caso del medio rural. Sin embargo, las diferencias entre un nivel de escolaridad y el siguiente, son un poco más significativas en la ciudad de México, así como también la diferencia existente entre las que tienen el menor y el mayor nivel de escolaridad. Esta alcanza ahora a 2.93 hijos nacidos vivos, mientras que en el medio rural era de 2.14 hijos nacidos vivos.

A nivel de grupos quinquenales de edad, la asociación negativa y uniforme entre la fecundidad y la escolaridad, más factible de esperar en el medio urbano, y conforme a la cual se formuló la primera parte de nuestra hipótesis de trabajo, se da principalmente a medida que se avanza en edad y que el número de entrevistadas es significativo. No obstante, todavía a este nivel no se está en condiciones de apoyar o refutar del todo esa parte de la hipótesis. Una excepción a lo expresado lo constituye el grupo 45-49 años para los dos últimos niveles de escolaridad. Se podría pensar que allí esté jugando cierto papel la conducta un tanto errática que muestra dicho grupo, y que por lo regular se trata de explicar en términos de olvido. Sin embargo, en este caso no se debe pensar en función de olvido, ya que se trata de las mujeres más preparadas. Más bien se considera que pueda estar ejerciendo su acción el reducido número de casos en esas casillas, o el grado de fidelidad o irrealidad de alguno de los valores.

3. Análisis de la relación entre la fecundidad y el grado de escolaridad, estandarizando por edad

En esta sección se analiza la influencia de las diferentes estructuras por edad de las madres, respecto al comportamiento de la relación entre la fecundidad y el grado de escolaridad. Esto se hace mediante un ejercicio de estandarización dado que las mujeres no se encuentran igualmente distribuidas, según su edad, en los diferentes niveles de escolaridad.

Para realizar dicho ejercicio, se sigue la metodología empleada por T. W. Pullum $(12,1977)$, en su trabajo Standarization, que aparece en el Boletín Técnico No 3 del Instituto Internacional de Estadística (véase el

7 A pesar de que la escolaridad viene desplegada en la encuesta en ocho categorías, la existencia de algunas casillas con ceros, y de otras, en donde el número de entrevistadas es muy reducido, obligó a efectuar agrupaciones en los grados de escolaridad. En ese sentido se unió el nivel de cero grados con 1-3 y la preparatoria con la universidad. 
Anexo al final). La estandarización permite estimar cuál sería el nivel de fecundidad (número medio de hijos nacidos vivos) según la escolaridad, bajo el supuesto de que todos los grupos tuvieran la misma estructura por edad tipo.

\section{Cuadro 9}

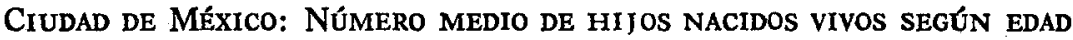
$Y$ GRADO DE ESCOLARIDAD DE LAS MADRES CASADAS Y CONVIVIENTES

\begin{tabular}{|c|c|c|c|c|c|c|c|}
\hline \multirow{2}{*}{ Grupos de edad } & \multicolumn{7}{|c|}{ Grado de escolaridad de las madres } \\
\hline & $0-3$ & $4-5$ & $\begin{array}{l}\text { Primaria } \\
\text { completa }\end{array}$ & Secundaria & $\begin{array}{l}\text { Preparatoria } \\
\text { y Universidad }\end{array}$ & $\begin{array}{l}\text { No } \\
\text { sabe }\end{array}$ & Total \\
\hline \multicolumn{8}{|l|}{$15-19$} \\
\hline Hijos & 28 & 9 & 15 & 8 & 0 & 0 & 60 \\
\hline Mujeres & 18 & 10 & 15 & 7 & 1 & 0 & 51 \\
\hline $\begin{array}{l}\text { Hijos por mujer } \\
20-24\end{array}$ & 1.56 & $0: 90$ & 1.00 & 1.14 & 0.00 & 0.00 & $1.1 \mathrm{~B}$ \\
\hline Hijos & 146. & $86^{\circ}$ & 88 & 97 & 29 & 0 & 446 \\
\hline Mujeres & 63 & 35 & 45 & 66 & 23 & 0 & 232 \\
\hline $\begin{array}{l}\text { Hijos por mujer } \\
25-29\end{array}$ & 2.32 & 2.46 & 1.96 & 1.47 & 1.20 & 0.00 & 1.92 \\
\hline Hijos & 338 & 94 & 179 & 209 & 57 & 3 & 880 \\
\hline Mujeres & 83 & 27 & 54 & 84 & 35 & 1 & 284 \\
\hline $\begin{array}{l}\text { Hijos por mujera } \\
30-34\end{array}$ & 4,07 & 3.48 & 3.31 & 2.49 & 1.63 & 3.00 & 3.10 \\
\hline Hijos & 575 & 164 & 200 & 212 & 78 & 0 & 1229 \\
\hline Mujeres & 108 & 31 & 50 & 63 & 33 & 1 & 286 \\
\hline $\begin{array}{l}\text { Hijos por mujera } \\
35-39\end{array}$ & 5.32 & 5.29 & 4.00 & 3.36 & 2.36 & 0.00 & 4.30 \\
\hline$\overline{\mathrm{HijOS}}$ & 583 & 220 & 200 & 284 & 65 & 10 & 1362 \\
\hline Mujeres & 89 & 40 & 41 & 63 & 19 & 1 & 253 \\
\hline $\begin{array}{l}\text { Hijos por mujeral } \\
40-43\end{array}$ & 6.55 & 5.50 & 4.88 & 4.51 & 3.42 & 10.00 & 5.38 \\
\hline$\overline{H i j o s}$ & 581 & 136 & 209 & 191 & 68 & 2 & 1187 \\
\hline Mujeres & 74 & 20 & 35 & 41 & 15 & 1 & 186 \\
\hline $\begin{array}{l}\text { Hijos por mujera } \\
45-49\end{array}$ & 7.85 & 6.80 & 5.97 & 4.66 & 4.53 & 2.00 & 6.38 \\
\hline Hijos & 506 & 138 & 1122 & 1104 & 59 & 44 & 93.3 \\
\hline Mujeres & 67 & 22 & 33 & 25 & 13 & 1 & 16.1 \\
\hline Hijos por mujeral & 7.55 & 6.27 & 3.70 & 4.16 & 4.54 & 4.00 & 5.80 \\
\hline Tota 1 & & & & & & & \\
\hline Hijos & 2757 & 847 & 1013 & 1105. & 356 & 19 & 6.097 \\
\hline Mujeres & 502 & 185 & 273 & 349 & 139 & 5 & 145.3 \\
\hline Hijos por mujeral & 5.49 & 4.58 & 3.71 & 3.17 & 2.56 & 3.80 & 4.20 \\
\hline
\end{tabular}

Fuente: Fase A de la Encuesta de Migración a la ciudad de México, 1970.

a Media aritmética.

Para llevar a efecto la estandarización, hay que tener presente que no exista interacción entre la edad y el grado de escolaridad, es decir, que para una edad particular el efecto de la escolaridad sobre la fecundidad se tiene que dar en el mismo sentido para todas las edades consideradas. Se puede observar si esa condición se cumple, mediante una gráfica con el número medio de hijos nacidos vivos según el grado de escolaridad, por grupos quinquenales de edad.

El comportamiento que sigue el número medio de hijos nacidos vivos se representa en la gráfica 1. Puede decirse que no existe interacción entre la edad y la escolaridad, pues con la excepción de cuatro puntos, en don- 
de la tendencia de las curvas cambia ligeramente, el efecto se manifiesta para todos los grupos de edades en el mismo sentido. Se considera que esos cuatro casos aislados, dos de los cuales se asocian con un número de entrevistados inferior a diez mujeres, no invalidan por sí solos la condición de no interacción, y en efecto, no impiden proceder a realizar la estandarización.

\section{Gráfica 1}

CIUdAd de México: Número MEdio de HIJOS NACIdos vivos NEGÚN EL GRado DE ESCOLARIDAD Y LA EDAD DE LAS MADRES CASADAS Y CONVIVIENTES, 1970

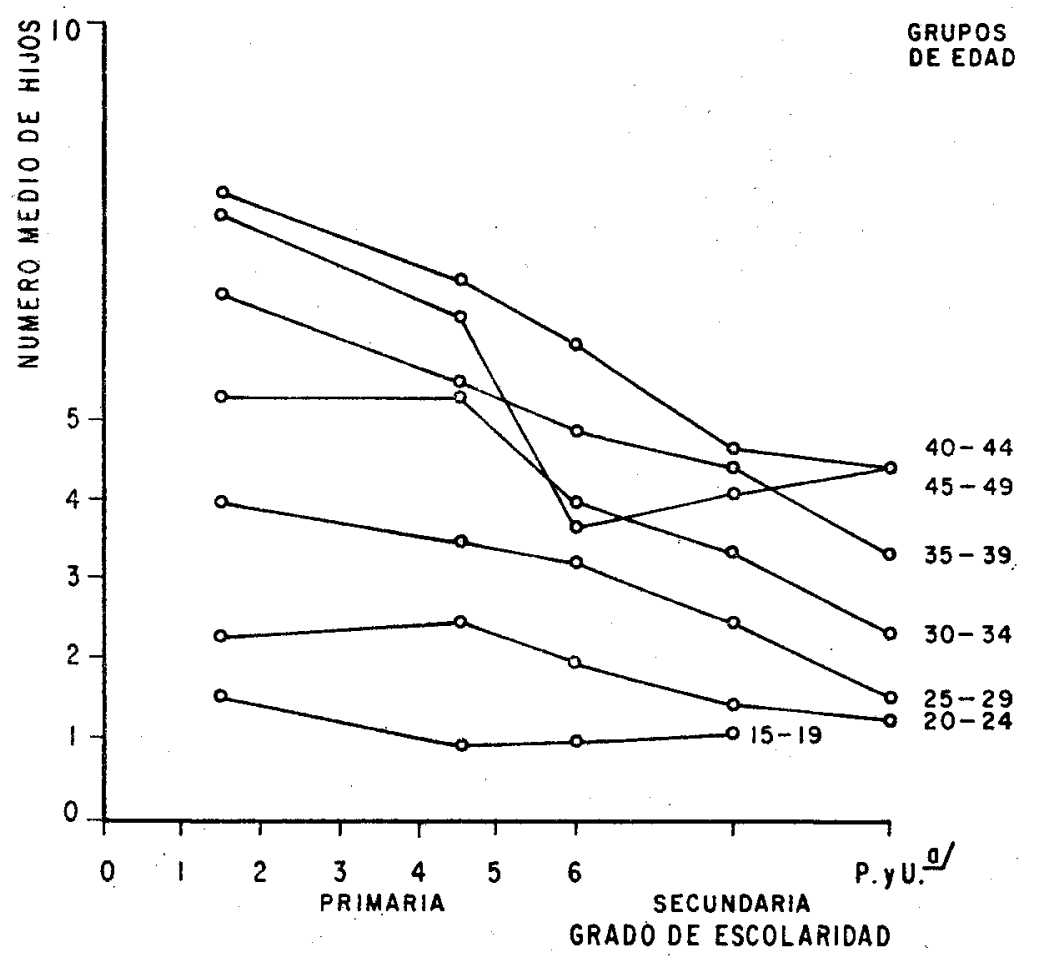

g/ Preparatorio y universidad

Con la finalidad de despejar la duda sobre el comportamiento del número medio de hijos nacidos vivos, para el grupo de edad 45-49 años en los dos últimos niveles de escolaridad, se procedió a realizar tres estandarizaciones: una en la que se tiene en cuenta dicho grupo, otra en la que se le incluye con el grupo 40-44 años, y otra en la que se elimina. Dado que el haber realizado tres estandarizaciones no condujo a encontrar resultados diversos, se comenta sólo la primera de ellas. A continuación apa- 
recen las "paridades medias" ${ }^{8}$ reales y estandarizadas según el nivel de escolaridad:

$$
E(0-3) \quad E(4-5) \quad E(P C)^{9} \quad E(S)^{9} \quad E(P \text { y } U)^{9}
$$

$\begin{array}{llllll}\text { Real } & 5.49 & 4.58 & 3.71 & 3.17 & 2.56 \\ \text { Estandarizada } & 5.25 & 4.67 & 3.81 & 3.27 & 2.66\end{array}$

Se puede apreciar que las paridades medias reales y estandarizadas, según nivel de escolaridad, no muestran mayores diferencias. En otras palabras, el hecho de haber adoptado una estructura por edad tipo, en este caso la del total de la población entrevistada casada y conviviente, no ha hecho cambiar en mayor grado la situación expresada por las paridades medias reales. Esto indica que el efecto de las diferentes estructuras por edad de las madres no es tan pronunciado en la ciudad de México. Recuérdese que al analizar el cuadro 8, se mencionó que en el Distrito Federal no sólo las mujeres más jóvenes (entre 15 y 34 años) alcanzaban los niveles más elevados de escolaridad, sino que también lo hacía una proporción significativa de mujeres de 35 y más años.

Veamos ahora el efecto neto ${ }^{10}$ del grado de escolaridad sobre las paridades medias reales y estandarizadas.

$\begin{array}{lccccc}\text { Efectos netos } & E(0-3) & E(4-5) & E(P C) & E(S) & E(P \text { y } U) \\ \begin{array}{cccc}\text { Efecto neto real } \\ \text { Efecto neto es- } \\ \text { tandarizado }\end{array} & 1.29 & 0.38 & -0.49 & -1.03 & -1.64 \\ & 1.09 & 0.51 & -0.35 & -0.89 & -1.50\end{array}$

Los efectos netos reales indican que en la ciudad de México, a medida que se adelanta en escolaridad, el número de hijos nacidos vivos es cada vez menor en relación a la paridad media, y que las mujeres que alcanzan la preparatoria o la universidad, tienen 1.64 hijos menos que la paridad media.

Al calcular el efecto neto estandarizado, es decir, aislada la acción de la edad, se observa que los nuevos valores para los diferentes niveles de escolaridad presentan ligeros cambios. Esto señala que en las diferencias observadas en el caso de los efectos netos reales, pesaba la acción de las diferentes estructuras por edad de las madres, y que una vez alejada dicha

8 Se entiende por paridad media, el número medio de hijos nacidos vivos, para determinado nivel de escolaridad.

9 $P C=$ primaria completa; $S=$ secundaria; y $P$ y $U$ preparatoria y universidad.

$10 \mathrm{Se}$ puede considerar el efecto neto como la acción definitiva del grado de escolaridad en la fecundidad, una vez eliminada la estructura por edad. Se obtiene al restar la paridad media total (real o estandarizada) de las paridades medias parciales, según los diversos grados de escolaridad. 
acción, la influncia del grado de escolaridad en la fecundidad se reduce ligeramente. Ahora, las mujeres con 0-3 grados de escolaridad van a tener 1.09 hijos más que la paridad media, y las mujeres que alcanzan el nivel más elevado de escolaridad tienen 1.50 hijos menos que la paridad media.

Es interesante anotar tres hechos: Primero, en la ciudad de México, los efectos netos estandarizados no muestran grandes diferencias con los efectos netos reales, situación que ya se había observado en el caso de las paridades medias. Ello conduce a reafirmar la posición que se había adoptado antes en el sentido de afirmar que la influencia de las estructuras por edad de las mujeres es menor que la observada en el caso del medio rural (véase, Zambrano Lupi, 1977). Segundo, aislada la influencia de la estructura por edad, se puede decir que en la ciudad de México se aprecia el cambio más significativo en el número medio de hijos nacidos vivos, una vez que se alcanza el nivel de escolaridad de la primaria completa. Es el momento que en relación al nivel anterior (4-5), se tienen 0.86 hijos nacidos y vivos menos. Tercero, aunque los niveles de escolaridad establecidos para la ciudad de México no son los mismos que se definieron para el medio rural, se puede afirmar que el efecto neto de la escolaridad, una vez que se alcanzan niveles de escolaridad más altos, es mayor en la ciudad de México.

Como conclusión de este apartado y al asociarlo con la primera parte de la hipótesis de este trabajo, se puede decir que la relación negativa y uniforme esperada en la ciudad de México entre la fecundidad y la escolaridad resultó cumplirse para los dos calificativos. Resultó "negativa" en el sentido de que una vez alejada la influencia de la estructura por edad, las mujeres que llegan al nivel de escolaridad más elevado tienen 1.59 hijos menos en la ciudad de México que en el medio rural; y adquirió mayor "uniformidad", porque también, realizada la estandarización (véase el cuadro 9), se puede apreciar un continuo descenso del número medio de hijos nacidos vivos.

4. Análisis de la relación entre la fecundidad y el grado de escolaridad en la ciudad de México, con referencia a la estratificación social de los individuos

En la segunda parte de la hipótesis del trabajo, se mencionó la posibilidad de que en la ciudad de México la relación fecundidad-escolaridad pudiera conocerse mejor mediante la introducción de alguna referencia a la posición social de los individuos.

Probar la bondad de esa parte de la hipótesis implica tener una variable que haga referencia a la estratificación social de los individuos. Esta variable se tomó del trabajo "Características socioeconómicas de las unidades domésticas del Distrito Federal", de Brígida García y Orlandina de Oliveira $(13,1977)$, quienes trabajaron con datos de la misma encuesta que sirvió de base a este trabajo. 
García y Oliveira construyen la variable denominada "posición social de los jefes de familias", para lo cual parten de la información disponible sobre la ocupación del cónyuge de las mujeres entrevistadas casadas y convivientes, lo que conduce a conocer los nexos ocupacionales que ligan el hogar, como unidad de reproducción, con los procesos productivos.

Se consideraron cinco criterios relacionados con la ubicación de los jefes de familia al interior de los sectores económicos. Dichos criterios fueron:

a) ocupación (profesionistas, técnicos, subprofesionistas, personal administrativo, trabajadores manuales calificados y no calificados, etc.);

b) posición en la ocupación: dependientes e independientes;

c) remuneración: remunerados y no remunerados;

d) personal empleado: ninguno; $1-4 ; 5$ y más;

e) propiedad o control: propietario o no propietario del local, del capital, de los medios de producción, etc.

A pesar de haberse considerado varios criterios, la diferenciación básica, al interior de los sectores, estuvo dada de manera fundamental según si el individuo vende o no su fuerza de trabajo (trabajadores asalariados dependientes y trabajadores independientes). La combinación de los cinco criterios mencionados, conduce al final a una clasificación de la posición social de los jefes de familia, que busca dar una visión aproximada de la manera en que dichos jefes de familia se insertan en las diferentes formas de organizar la producción que existen en la ciudad de Méxici. Dicha clasificación aparece a continuación:

a) no trabajó y no tiene empleo;

b) empleadores (trabajadores independientes) con más de cinco empleados remunerados;

c) empleadores (trabajadores independientes), con uno a cuatro empleados remunerados;

d) autónomos no manuales (trabajadores independientes), sin personal remunerado;

e) autónomos manuales (trabajadores independientes), sin personal remunerado.

f) empleados no manuales (trabajadores dependientes), con personal bajo sus órdenes.

g) empleados no manuales (trabajadores dependientes), sin personal bajo sus órdenes;

h) trabajadores manuales (trabajadores dependientes), calificados;

i) trabajadores manuales (trabajadores dependientes), no calificados,

a) Análisis de la relación entre la posición social de los jefes de familia, la edad de las madres y la fecundidad. En el cuadro 10, aparece el 
número medio de hijos nacidos vivos según las condiciones citadas. A nivel de grupos de edad, se carece de elementos suficientes para establecer conclusiones al respecto dado que existen algunas casillas con ceros y otras con un número muy reducido de casos.

En función de la posición social de los jefes de familia, los resultados muestran algunas características no acordes con lo comúnmente esperado, es decir, la tendencia inversa entre el prestigio de la ocupación y la fecundidad, no se aprecia de una forma tan gradual, como de manera ordinaria se caracteriza en términos teóricos.

Lo primero que llama la atención es que, en términos generales, el número medio de hijos nacidos vivos de las mujeres casadas y convivientes cuyos cónyuges se encuentran catalogados dentro de las actividades independientes, es superior (4.37) al número medio de hijos nacidos vivos de las mujeres casadas y convivientes cuyos cónyuges están comprendidos dentro de las actividades dependientes (3.92). Aunque la diferencia es de sólo 0.45 hijos, ocurre que para la ciudad de México se da de una manera contraria a lo esperado.

Si se observa el número medio de hijos nacidos vivos para cada una de las posiciones sociales de los jefes de familia, también se encuentran situaciones opuestas a lo usual. De manera concreta, resulta que los empleados constituyen en la ciudad de México, junto con los empleadores con más de cinco empleados remunerados, la posición social de los jefes de familia, en donde el número medio de hijos nacidos vivos es menor, con una diferencia de más de un hijo nacido vivo en relación a los autónomos no manuales, que comprende el grupo integrado, entre otras personas, por los profesionistas, técnicos, subprofesionistas, etc., quienes se supone han alcanzado los niveles de escolaridad más elevados. También se puede apreciar que dentro del grupo de los autónomos manuales (artesanos), es donde el número medio de hijos nacidos vivos es mayor, incluso dicha cifra es superior a la observada en el caso de los trabajadores manuales calificados y no calificados.

Intentar una explicación de esos hallazgos, sin tener suficientes elementos de juicio, sólo conduce a caer en especulaciones. A pesar de ello, se consideran algunas razones que de una $u$ otra manera tienen alguna influencia y, como se ha hecho frecuente, se apela al argumento económico. De esta manera se tiene la oportunidad de probar si dicho razonamiento es o no válido en el caso de la ciudad de México.

El argumento económico, Becker $(14,1960)$, Leibenstein $(15,16$, $1975,1977)$, Bultato $(17,1977)$, etc., para sólo citar tres autores, en términos generales, " predice que si en algún sentido el precio de los niños llega a un nivel suficientemente alto, la fecundidad descenderá, a pesar del aumento en los ingresos" (16; p. 2). Es decir, la teoría económica de la fecundidad coloca a los individuos ante la disyuntiva de postergar el matrimonio y limitar la fecundidad, para permitirse el acceso al mercado 
Cuadro 10

Ciudad de MÉxico: Número de hijos Nacidos vivos de las Mujeres CASADAS Y CONVIVIENTES, SEGÚN LA POSICIÓN SOCIAL DE LOS JEFES DE FAMILIA Y GRUPOS DE EDAD DE LAS MADRES

\begin{tabular}{|c|c|c|c|c|c|c|c|c|}
\hline \multirow{2}{*}{$\begin{array}{l}\text { Posicion social de } \\
\text { zos jefes de familia }\end{array}$} & \multicolumn{7}{|c|}{ Edad de las madres } & \multirow{2}{*}{ Total } \\
\hline & $15-19$ & $20-24$ & $25-29$ & $30-34$ & $35-39$ & $40-44$ & $45-49$ & \\
\hline \multicolumn{9}{|l|}{$\begin{array}{l}\text { No- trabajt y no tiene } \\
\text { enpleo }\end{array}$} \\
\hline Hijos & o & & 20 & 18 & 33 & 45 & 53 & 169 \\
\hline Mujeres & 1 & - & 5 & 5 & 6 & 6 & 7 & 30 \\
\hline Hijos por rujer & 0.00 & - & 4.00 & 3.60 & 5.50 & 7.50 & 7.57 & 5.63 \\
\hline \multicolumn{9}{|c|}{ Empleadores $(5 \circ$ o măs $)$ a/ } \\
\hline & 1 & 8 & 17 & 39 & 26 & 24 & 19 & 234 \\
\hline Mujeres & 1 & 3. & 9 & 11 & 8 & 6 & 5 & 43 \\
\hline Hijos por mujer & 1.00 & 2.67 & 1.89 & 3.54 & 3.25 & 4.00 & 3.80 & 3.12 \\
\hline \multicolumn{9}{|l|}{ Smpleadores $(1 ; 4) \underline{b}$} \\
\hline Hijos & 2 & 15 & 37 & 60 & 136 & 93 & 30 & 373 \\
\hline Mujeres & 3 & 9 & 14 & 19 & 21 & 14 & 5 & 85 \\
\hline Hijos por mujer & 0.67 & 1.67 & 2.64 & 3.16 & 6.48 & 5.64 & 6.00 & 4.39 \\
\hline \multicolumn{9}{|c|}{ Autônomos no manuales $\underline{\text { f }}$} \\
\hline Hijos & 5 & 25 & 45 & 103 & 159. & 53 & 98 & 489 \\
\hline Mujeres & $\mathbf{3}$ & 12 & 15 & 19 & 26 & 9 & 20 & 104 \\
\hline Hijos por mujer & 1.67 & 2.17 & 3.00 & 5.42 & 6.11 & 5.89 & 4.90 & 4.70 \\
\hline \multicolumn{9}{|l|}{ Autônomos manuales } \\
\hline Hijos & - & 15 & 30 & 55 & 45 & 68 & 56 & 279 \\
\hline Mujeres & - & 7 & 9 & 11 & 7 & 10 & 9 & 5.3 \\
\hline Hijos por mujer & - & 2.14 & 3.33 & 5.91 & 5.43 & 6.80 & 6.22 & 5.26 \\
\hline \multicolumn{9}{|c|}{$\begin{array}{l}\text { Empleados no manuales con } \\
\text { personaldf }\end{array}$} \\
\hline Hijos & 7 & so & 110 & 122 & 75. & 147 & 122 & 633 \\
\hline Mujeres & 4 & 28 & 47 & 37 & 25 & 27 & 27 & 195 \\
\hline Hijos por mujer & 1.75 & 1.78 & 2.34 & 3.30 & 3.00 & 5.44 & 4.52 & 3.25 \\
\hline \multicolumn{9}{|c|}{$\begin{array}{l}\text { Empleados no manuales sin } \\
\text { personalef }\end{array}$} \\
\hline $\mathrm{Hijos}$ & 9 & 83 & 123 & 143 & 191 & 103 & 81 & 733 \\
\hline Mujeres & 6 & 5 & 49 & 42 & 36 & 22 & 20 & 230 \\
\hline Hijos por mujer & 1.50 & 1.51 & 2.61 & 3.40 & 5.31 & 4.68 & 4.05 & 3.19 \\
\hline \multicolumn{9}{|c|}{ Trabajadores calificados $\underline{f \prime}$} \\
\hline Hijos & 13 & 108 & 259 & 312 & 432 & 277 & 192 & 1593 \\
\hline Mujeres & 16 & 52 & 71 & 72 & 75 & 45 & 29 & 360 \\
\hline Hijos por mujer & 0.81 . & 2.08 & 3.65 & 4.33 & 5.76 & 6.16 & 5.62 & 4.42 \\
\hline \multicolumn{9}{|c|}{ Trabajadores no calificados fl } \\
\hline Hijos & 23 & 133 & 235 & 353 & 262 & 369 & 282 & 1657 \\
\hline Mujeres & 17 & 62 & 64 & 67 & 48 & 46 & 39 & 343 \\
\hline Hijos por mujer & 1.35 & 2.14 & 3.67 & 5.27 & 5.46 & 8.02 & 7.23 & 4.83 \\
\hline \multicolumn{9}{|l|}{ No se aplica } \\
\hline Hijos & - & B & 4 & 14 & 3 & B & - & 37 \\
\hline Mujeres & - & 4 & 1 & 3 & 1 & 1 & - & 10 \\
\hline Hijos por mujer & - & 2.00 & 4.00 & 4.67 & 3.00 & 8.00 & - & 3.70 \\
\hline \multicolumn{9}{|l|}{ Total } \\
\hline Hijos & 60 & 446 & 880 & 1229 & 1362 & 1187 & 933 & $6 \quad 097$ \\
\hline Mujeres & 51 & 232 & 284 & 285. & 253 & 195 & 161 & 1453 \\
\hline Hijos por mujer & 1.18 & 1.92 & 3.10 & 4.30 & 5.38 & 6.38 & 5.79 & 4.20 \\
\hline
\end{tabular}

Fuente: Fase A de la Encuesta de Migración a la ciudad de México, 1970.

a Trabajadores independientes con más de cinco empleados remunerados.

b Trabajadores independientes con 1 a 4 empleados remunerados.

c Trabajadores independientes sin personal bajo sus órdenes.

d Trabajadores dependientes con personal bajo sus órdenes.

- Trabajadores dependientes sin personal bajo sus órdenes.

Trabajadores manuales dependientes. 
de bienes suntuarios, lo que lleva, en otras palabras, a optar entre los hijos y la inversión.

Dicha teoría se ha venido sustentando en un gran número de estudios, los cuales tanto en el pasado como en el presente asocian el descenso de la fecundidad con diferentes variables socioeconómicas. Al igual que para Leibenstein $(16$, p. 3$)$, "esos macro-estudios, en esencia, generalmente tratan de tendencias estadísticas generales más que del examen de procesos de tomas de decisión en micro unidades", y asocian el descenso en la fecundidad con una mayor educación de las mujeres, una mayor participación de éstas en la mano de obra, una mayor movilidad ocupacional y social con elevados índices de urbanización, con costos elevados de educación infantil, con la desaparición del sistema familiar numeroso, con una mayor disponibilidad de información y de técnicas anticonceptivas.

Las orientaciones que sigue dicha teoría para desarrollar un modelo del descenso en la fecundidad son múltiples. Entre las principales posiciones, Leibenstein alega que el número de hijos deseados depende de las utilidades relativas de los beneficios y de los costos que los hijos representan para los padres, mientras los gastos por cada niño están determinados por la posición socioeconómica de sus padres. Becker, en su posición original, al considerar sólo el "valor de consumo" de los niños, aduce que la decisión que determina el tamaño de la familia es similar a la que se usaba para determinar la compra de bienes de consumo duraderos. Según esto, indicó que los grupos de mayores ingresos compran, por decirlo asi, niños de mejor calidad y de hecho gastan más por cada niño. En un ensayo posterior, Becker cambió su posición original e indicó que debido a que los gastos por cada niño eran iguales, el resultado sería un precio mayor para los ricos que para los pobres.

Aunque existe otra serie de orientaciones al respecto, se considera que las formulaciones anteriores permiten vislumbrar los argumentos de la teoría económica de la fecundidad en el caso de la ciudad de México.

Conforme a nuestro análisis, al introducir el argumento económico, se encontró que su validez sólo sería aproximada en el caso de los empleados. Recuérdese que es este grupo el que tiene el menor número medio de hijos nacidos vivos. En términos generales, los empleados constituyen un conjunto heterogéneo, integrado por aquellos asalariados englobados en una serie de ocupaciones no manuales existentes en los diversos sectores de la actividad económica, pero que con mayor regularidad median en las operaciones propias de la burocracia, en donde por lo general están sujetos a presiones de movilidad social; las condiciones propias de su situación hacen posible pensar que retrasan un poco el matrimonio y que una vez casados posterguen el advenimiento de los hijos, dadas sus mayores aspiraciones en el sentido mencionado.

Parece evidenciarse en el caso de los empleados la postulación de Leibenstein en el sentido de que los gastos según el número de hijos están 
determinados por la posición socioeconómica de los padres. Como aquí por el momento no se puede probar lo antes expresado, se indica sólo a nivel de hipótesis explicativa, para el caso mencionado.

Conforme a este tipo de especulaciones, sería lógico esperar que dentro de las posiciones sociales de los jefes de familia de menor prestigio (autónomos manuales y trabajadores manuales calificados y no calificados), se observara un menor número medio de hijos nacidos vivos, dada la situación de estrechez económica en que viven esos grupos. Sin embargo, no sucede de esa manera. Por el contrario, son las categorías de posición social en donde el número medio de hijos nacidos vivos es mayor. Constituye este caso uno de tantos, en donde entran en juego las debilidades del argumento económico.

Si se observa la posición de los empleadores, se percibe que su situación es ambigua. Por una parte, los empleadores con más de cinco empleados remunerados tienen un reducido número medio de hijos (3.12), y son estas personas (grupo formado de manera principal por patrones y trabajadores por cuenta propia, grandes y medianos empresarios, etc.) quienes movilizan mayor capital, y supuestamente quienes estarían menos presionados ante una posición en donde deban decidir entre invertir en hijos o invertir en bienes, pero podría ser que en sus decisiones pese más lo relacionado a la calidad de los hijos. Parece cobrar validez para este tipo de posición social el argumento de Becker, cuando dice que los grupos de mayores ingresos compran niños de mejor calidad, $y$ de hecho gastan más por cada niño.

Por otro lado, los empleadores con uno a cuatro empleados remunerados, tienen un número medio de 4.39 hijos nacidos vivos, cifra elevada si se piensa que constituyen un grupo en donde debería privar la conducta a escoger entre los hijos y la inversión en bienes, dado que se trata de estratos en ascenso, es decir, de grupos sociales con grandes posibilidades de movilidad social. Por último, llama bastante la atención que los autónomos no manuales tengan un número medio de 4.70 hijos nacidos vivos.

b) Análisis de la relación entre la fecundidad y el grado de escolaridad conforme a la posición social. Hecho el análisis de la relación posición social-fecundidad en función de la edad de las madres, se considera ahora en relación a los niveles de escolaridad.

A fin de contar con suficiente número de casos en relación a la edad, se establecieron tres grandes grupos de edades: 15-19 y 20-24; 25-29 y 30-34; y 35 años y más. En lo concerniente a las posiciones sociales de los jefes de familia, después de varios intentos se procedió a agrupar dichas posiciones en tres conjuntos: En el primero se agruparon todos los empleadores con los autónomos no manuales; en el segundo a los empleados, independientemente o no de que tengan personal bajo sus órdenes; y en el tercero, se asociaron los autónomos manuales con los trabajadores manuales calificados y no calificados. Dicha agrupación se hizo en atención a las siguientes razones: 
i) Al observar en general el número medio de hijos nacidos vivos para cada una de las posiciones sociales y cada uno de los grupos quinquenales de edad, se apreció que, aún cuando no se pueda generalizar para todos los grupos de edades, los empleados, al margen de que tengan o no personal bajo sus órdenes, tienen en términos generales un número medio de hijos nacidos vivos muy similar. Los autónomos manuales se asemejan mucho, en cuanto al número medio de hijos nacidos vivos, a los trabajadores manuales calificados y no calificados; de ahí que se agruparan en un solo conjunto. Por último, aunque las semejanzas en el número medio de hijos, entre los empleadores y los autónomos no manuales, son menores que en los casos anteriores, de manera principal entre los empleadores con cinco y más empleados remunerados y los que tienen entre uno y cuatro empleados remunerados, se decidió agruparlos. La otra solución posible (dejar solos a los empleadores con más de cinco empleados remunerados), presentaba el inconveniente de contar con un número muy reducido de casos.

ii) En segundo lugar, se consideró que la agrupación tal como se hizo, conducía a una posición de estratificación social hasta cierto punto aproximada. Los integrantes de cada uno de los grupos presentan características, en unos casos de tipo socioeconómico y en otros de tipo ocupacional, más o menos semejantes.

En tal sentido, si se pensó en términos de una estratificación piramidal, es lícito suponer que los empleadores, dado que en sus condiciones socioeconómicas se asemejan más a los autónomos no manuales, pueden constituir ambos grupos una aproximación a una clase alta o a lo sumo media-alta.

Los empleados, con y sin personal bajo sus órdenes, al constituir el grueso de los asalariados, concentrados de manera principal en las labores de la burocracia, constituyen un grupo que a pesar de no ser homogéneo económicamente y de mostrar discrepancias en relación a su grado de subordinación dentro de la estratificación burocrática, presentan el denominador común de estar insertos sobre todo en el sector servicios. Por ello, están expuestos a una serie de valores y culturas urbanas, tal vez pueda decirse "modernas", muy semejantes. Por último, se considera que las diferencias socioeconómicas entre los autónomos manuales y los trabajadores manuales calificados y no calificados no son tan grandes como para que impidan agruparlos juntos.

Se es consciente de que las agrupaciones realizadas no son perfectas y dejan a un lado una serie de condicionantes. En ningún momento se pretende que se puedan generalizar, ni que sean las más representativas socioeconómicamente, pero para los efectos de este trabajo parecieron las más aproximadas, una vez que se combinaron el comportamiento reproductivo y las características socioeconómicas.

En cuanto a la escolaridad, se agregaron los niveles 0-3 y 4-5, la primaria completa se dejó en su forma original y se agruparon la secundaria, 
la preparatoria y la universidad. ${ }^{11} \mathrm{La}$ unión de los dos primeros grupos se debió a que al sumar un mayor número de entrevistadas, se podía obtener una visión más precisa que si se mantenían dichos niveles por separado. Por igual razón se agruparon la secundaria, la preparatoria y la universidad.

El comportamiento que sigue el número medio de hijos nacidos vivos, hechas las agrupaciones, se puede observar en el cuadro 11, y la distribución proporcional de las mujeres casadas y convivientes, según las mismas condiciones, aparece en el cuadro 12.

Cuatro hechos resaltan una vez realizada la reagrupación mencionada. El primero es que el número medio de hijos nacidos vivos continúa siendo inferior, independientemente de la edad, dentro del grupo de los empleados (renglones y columnas de totales). Una vez más este resultado respecto a la ciudad de México, genera dudas sobre la renombrada asociación teórica entre el prestigio de la ocupación y el nivel de la fecundidad; aun cuando se debe tener presente que aún no se ha indagado sobre la manera como la diferente estructura por edad de las madres pueda estar ejerciendo su influencia.

El segundo hecho es que las diferencias en el número medio de hijos nacidos vivos existentes entre los empleados y el grupo formado por los empleadores y autónomos no manuales, se mantienen en todos los grupos de edad (columnas de totales). De manera concreta, las diferencias son de 0.24 hijos a favor de los empleadores y autónomos no manuales en el grupo de edad 15-19 y 20-24 años; de 0.65 hijos en el grupo 25-29 y 30-34 años; y de 1.06 hijos en el grupo 35 años y más. Esto señala que la situación se da tanto para las mujeres más jóvenes como para las que han cumplido parte de su vida reproductiva y para las que han terminado su ciclo fecundo.

El tercer hecho es, como era de esperarse, que el conjunto formado por los autónomos no manuales y los trabajadores manuales calificados y no calificados, continúa presentando en casi todas las edades el número medio de hijos nacidos vivos más elevado.

Por último, es interesante advertir que la reagrupación de los niveles de escolaridad surtió efecto, en el sentido de que el comportamiento seguido por el número medio de hijos nacidos vivos, dentro de cada uno de los conjuntos de posición social de los jefes de familia, se aproxima al esperado; es decir, a mayor nivel de escolaridad, el número medio de hijos nacidos vivos disminuye de manera continua. Una excepción al respecto la constituyen dos casos dentro del grupo de los empleadores y autónomos no manuales, pero en dicha situación juega un papel importante el reducido número de entrevistados, que aún persiste a pesar de haber hecho nuevas agrupaciones.

11 La notación $S, P$ y $U$ significan secundaria, preparatoria y universidad, respectivamente. 


\section{Cuadro 11}

NÚMERO MEdIO dE HIJOS NACIDOS VIVOS DE LAS MU JERES CASADAS Y CONVIVIENTES, SEGÚN GRUPO DE EDAD $Y$ NIVELES DE ESCOLARIDAD EN LOS DIFERENTES GRUPOS DE POSICIÓN SOCIAL DE LOS JEFES DE FAMILIA

\begin{tabular}{|c|c|c|c|c|c|c|c|c|c|c|c|c|c|c|c|c|c|}
\hline \multirow{3}{*}{ Grupos de edad } & \multicolumn{16}{|c|}{ Posición social y grado de escolaridad-? } & \multirow{3}{*}{ Total } \\
\hline & \multicolumn{4}{|c|}{$\begin{array}{l}\text { Enpleadores y autonomos } \\
\text { no manuales }\end{array}$} & \multicolumn{4}{|c|}{ Empleados } & \multicolumn{4}{|c|}{$\begin{array}{l}\text { Aut } \text { tronos manuales y } \\
\text { trabajadores manuales }\end{array}$} & \multicolumn{4}{|c|}{$\begin{array}{l}\text { No trabajan, no tienen } \\
\text { empleo y no se aplica }\end{array}$} & \\
\hline & $0-5$ & $\mathrm{PC}$ & $S P$ y $U$ & Total & $0-5$ & $\mathrm{PC}$ & $S P$ y $U$ & TotaI & $0-5$ & PC & $S P$ y $U$ & Total & $0-5$ & PC & $S P$ Y U & Total & \\
\hline \multicolumn{18}{|l|}{$15-19 \times 20-24$} \\
\hline Hijos & 22 & 11 & 24 & 57 & 48 & 30 & 71 & 149 & 195 & 59 & 38 & 292 & 4 & 3 & 1 & 8 & 506 \\
\hline Mujeres & 10 & 9 & 12 & 31 & 21 & 15 & 57. & 93 & 93 & 34 & 27 & 154 & 2 & 2 & 1 & $s$ & 283 \\
\hline Hijos por mujer & 2.20 & 1.22 & 2.00 & 1.84 & 2.29 & 2.00 & 1.25 & 1.60 & 2.10 & 1.73 & 1.41 & 1.90 & 2.00 & 1.50 & 1.00 & 1.60 & 1.79 \\
\hline \multicolumn{18}{|l|}{$25-29$ y $30-34$} \\
\hline Hijos & 114 & 72 & 115 & 301 & 152 & 94 & 252 & 498 & 874 & 201 & 176 & 1251 & 31 & 12 & 13 & 56 & 2106 \\
\hline Mujere's & 25 & 17 & 44 & 86 & 31 & 29 & 115 & 175 & 186 & 55 & 52 & 293. & 7 & 3 & 4 & 24 & 568 \\
\hline Hijos por mujer & 4.56 & 4.23 & 2.61 & 3.50 & 4.90 & 3.24 & 2.19 & 2.85 & 4.70 & 3.65 & 3.38 & 4.27 & 4.43 & 4.00 & 3.25 & 4.00 & 3.71 \\
\hline \multicolumn{18}{|l|}{35 y más } \\
\hline Hijos & 349 & 83 & 200 & 632 & 156 & 175 & 388 & 719 & 1566 & 248 & 159 & 1973 & 93 & 25 & 24 & 142 & 3466 \\
\hline Mujeres & 51 & 18 & 43 & 112 & 27 & 39 & 91 & 157 & 222 & 47 & 38 & 307 & 12 & 5 & 4 & 21 & 697 \\
\hline Hijos por mujer & 6.84 & 4.61 & 4.65 & 5.64 & 5.78 & 4.49 & 4.26 & 4.58 & 7.05 & 5.25 & 4.18 & 6.43 & 7.75 & 5.00 & 6.00 & 6.76 & 5.81 \\
\hline \multicolumn{18}{|l|}{ Tota1 } \\
\hline$\overline{\text { Hijos }}$ & 485 & 166 & 339 & 990 & 356 & 299 & 711 & 1366 & 2635 & 508 & 373 & 3526 & 128 & 40 & 38 & 206 & $60782^{\prime}$ \\
\hline Mujeres & 86 & 44 & 99 & 229 & 79 & 83 & 263 & 425 & 501 & 136 & 117 & 754 & 21 & 10 & 9 & 40 & $144 E^{-b}$ \\
\hline Hijos por mujex & 5.64 & 3.77 & 3.42 & 4.32 & 4.51 & 3.60 & 2.70 & 3.21 & 5.26 & 3.73 & 3.19 & 4.66 & 6.09 & 4.00 & 4.22 & 5.15 & 4.20 \\
\hline
\end{tabular}

a Grado de escolaridad: 0-5: hasta cinco años de primaria; PC: primaria completa; SP y U: secundaria, preparatoria y universidad.

b Las ligeras diferencias observadas en el total respecto al de otros cuadros se deben a que se eliminaron las mujeres de la categoría "no sabe". 


\section{Cuadro 12}

Distribución DE LAS MUjeres CASADAS Y CONVIVIENTES ENTREVISTADAS, SEGÚN EDAD Y NiVEL DE ESCOLARIDAD EN CADA GRUPO DE POSICIÓN SOCIAL DE LOS JEFES DE FAMILIA

\begin{tabular}{|c|c|c|c|c|c|c|c|c|c|c|c|c|c|c|}
\hline \multirow{3}{*}{$\begin{array}{l}\text { Grupos de } \\
\text { edad }\end{array}$} & \multicolumn{12}{|c|}{ Yosición social y grado de escolaridad-al } & \multirow{3}{*}{$\mathrm{NT}^{\mathrm{b}}=$} & \multirow{3}{*}{ Total } \\
\hline & \multicolumn{4}{|c|}{$\begin{array}{l}\text { Emp leadores y autónomos } \\
\text { no manuales }\end{array}$} & \multicolumn{4}{|c|}{ Empleados } & \multicolumn{4}{|c|}{$\begin{array}{l}\text { Autônomos manuales y traba- } \\
\text { jadores manuales }\end{array}$} & & \\
\hline & 0.5 & $\mathrm{PC}$ & $S P$ y $U$ & Total & $0-5$ & PC & SP y $U$ & Total & $0-5$ & PC & SP y U & Total & & \\
\hline \multicolumn{15}{|c|}{$15-19$ y $20-24$} \\
\hline $\begin{array}{l}\text { Mujeres } \\
\text { o renglon of } \\
\text { o columna }\end{array}$ & $\begin{array}{l}10 . \\
32.3 \\
11.6\end{array}$ & $\begin{array}{r}9 \\
29.0 \\
20.6\end{array}$ & $\begin{array}{l}12 \\
38.7 \\
12.2\end{array}$ & $\begin{array}{r}31 \\
100.0 \\
13.5\end{array}$ & $\begin{array}{c}21 \\
22.6 \\
26.5\end{array}$ & $\begin{array}{l}16 \\
16.1 \\
18.1\end{array}$ & $\begin{array}{l}57 \\
61.3 \\
21.7\end{array}$ & $\begin{array}{c}93 \\
100.0 \\
21.9\end{array}$ & $\begin{array}{c}93 \\
60: 4 \\
18.6\end{array}$ & $\begin{array}{l}34 \\
22.1 \\
25.0\end{array}$ & $\begin{array}{c}27 \\
17.6 \\
23.1\end{array}$ & $\begin{array}{c}154 . \\
100.0^{\circ} \\
20.4\end{array}$ & $\begin{array}{l}6 \\
-\end{array}$ & $\begin{array}{r}283 \\
13.5\end{array}$ \\
\hline \multicolumn{15}{|c|}{$25-29 \times 30-34$} \\
\hline 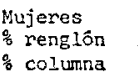 & $\begin{array}{l}25 \\
29.0 \\
29.1\end{array}$ & $\begin{array}{l}17 \\
19.8 \\
38.6\end{array}$ & $\begin{array}{c}4.4 \\
51.2 \\
44.6\end{array}$ & $\begin{array}{r}86 \\
100.0 \\
37.6\end{array}$ & $\begin{array}{c}31 \\
17.7 \\
39.2\end{array}$ & $\begin{array}{c}29 \\
16.6 \\
34.9\end{array}$ & $\begin{array}{l}115 \\
65.7 \\
43.7\end{array}$ & $\begin{array}{c}175 \\
100.0 \\
41.2\end{array}$ & $\begin{array}{l}186 \\
63.5 \\
37.1\end{array}$ & $\begin{array}{c}55 \\
18.8 \\
40.4\end{array}$ & $\begin{array}{c}52 \\
17.7 \\
44.4\end{array}$ & $\begin{array}{c}293 \\
100: 0 \\
38.9\end{array}$ & 16 & $\begin{array}{c}570 \\
30.2\end{array}$ \\
\hline \multicolumn{15}{|l|}{$35 \mathrm{y}$ mâs } \\
\hline $\begin{array}{l}\text { Mujeres } \\
\text { o rengión } \\
\text { \% columna }\end{array}$ & $\begin{array}{c}51 \\
45.5 \\
59.3\end{array}$ & $\begin{array}{l}18 \\
16.1 \\
40.9\end{array}$ & $\begin{array}{l}43 \\
38.4 \\
43.4\end{array}$ & $\begin{array}{r}112 \\
100.0 \\
48.9\end{array}$ & $\begin{array}{c}27 \\
17.2 \\
34.2\end{array}$ & $\begin{array}{c}39 \\
24.8 \\
47.0\end{array}$ & $\begin{array}{c}91 \\
58.0 \\
34.6\end{array}$ & $\begin{array}{c}157 \\
100.0 \\
36.9\end{array}$ & $\begin{array}{l}222 \\
72.3 \\
44.3\end{array}$ & $\begin{array}{l}47 \\
15.3 \\
34.3\end{array}$ & $\begin{array}{c}38 \\
12.4 \\
32.5\end{array}$ & $\begin{array}{c}307 \\
100.0 \\
40.7\end{array}$ & $\begin{array}{l}24 \\
-\end{array}$ & $\begin{array}{c}620 \\
-.3 \\
41.3\end{array}$ \\
\hline \multicolumn{15}{|l|}{ Total } \\
\hline $\begin{array}{l}\text { Mujeres } \\
\text { orengión } \\
\text { of columna }\end{array}$ & $\begin{array}{r}86 \\
37.3 \\
100.0\end{array}$ & $\begin{array}{c}44 \\
19.2 \\
100.0\end{array}$ & $\begin{array}{r}99 \\
43.2 \\
100.0\end{array}$ & $\begin{array}{r}229 \\
100.0 \\
100.0\end{array}$ & $\begin{array}{r}79 \\
19.5 \\
100.0\end{array}$ & $\begin{array}{r}83 \\
19.5 \\
100.0\end{array}$ & $\begin{array}{c}263 \\
61.9 \\
100.0\end{array}$ & $\begin{array}{c}425 \\
120.0 \\
100.0\end{array}$ & $\begin{array}{c}501 \\
65.5 \\
100.0\end{array}$ & $\begin{array}{r}136 \\
138.0 \\
100.0\end{array}$ & $\begin{array}{r}117 \\
15.5 \\
100.0\end{array}$ & $\begin{array}{c}754 \\
100.0 \\
100.0\end{array}$ & $=$ & $\begin{array}{l}1453 \\
100.0\end{array}$ \\
\hline
\end{tabular}

FUENTE: Encuesta de Migración a la ciudad de México, 1970.

a 0-5: hasta cinco años de educación primaria; PC: primaria completa; SP y U: secundaria, preparatoria y universidad.

b No trabajan, no tienen empleo o no responden.

c Respecto al total de cada posición social.

d Respecto al total de cada grado de escolaridad. 
$\mathrm{Al}$ analizar la distribución de las mujeres entrevistadas casadas y convivientes y conformes a las cifras del cuadro 12 , se puede decir que se aprecian tres situaciones particulares, las cuales se asocian con los diferentes conjuntos de posición social. Dentro del grupo formado por los empleadores y autónomos no manuales, en términos medios se ve que las mujeres se concentran en mayor proporción dentro del primero y el último nivel de escolaridad, siendo incluso mayor el valor de dicha distribución en el segundo de los casos (renglones totales). En atención a los grupos de edad y el nivel de escolaridad, esta distribución acusa mayores variaciones, aunque por lo general se conserva la tendencia indicada, lo cual expresa dos hechos: $a$ ) en la ciudad de México, dentro de ese grupo de posición social, al tener las mujeres mayores posibilidades de estudio, $\mathrm{y}$ al ofrecer el sistema educativo en dicha ciudad una gama más variada y completa de oportunidades de aprendizaje, las mujeres aparecen distribuidas según su edad y su escolaridad de una forma más variada; y $b$ ) más que la razón antes expuesta, se debe tener presente que la proporción de mujeres en esa posición social (que en términos generales alcanzan en la ciudad de México el nivel más elevado de escolaridad), es bastante significativa.

Dentro del conjunto integrado por los empleados, en términos generales, la situación varía en relación a la descrita antes. Cabe destacar dos aspectos: El primero es que en términos medios, la proporción de mujeres que alcanzan el nivel más elevado de escolaridad $(61.9 \%)$, es mayor aún que la observada en el caso de los empleadores y autónomos no manuales (43.2\%) (renglones de totales). Esto indica que no es entonces un hecho fortuito que el número medio de hijos nacidos vivos dentro de este grupo de posición social sea el más bajo, sino que en la ciudad de México las mujeres casadas y convivientes, cuyos cónyuges son empleados, son quienes en mayor proporción arriban a un nivel de escolaridad más alto, independientemente de la edad. Esta razón, unida a otros factores que también pueden ejercer su influencia, parece justificar el comportamiento reproductivo dentro del conjunto de los empleados. El segundo aspecto que se aprecia es que, en relación a la edad y a los niveles de escolaridad, las mujeres más jóvenes en este grupo alcanzan ahora mayor importancia relativa, sobre todo en los dos últimos niveles de escolaridad (columna de porcientos).

Por último, dentro de los autónomos manuales y los trabajadores manuales, se aprecia una situación, podría decirse, inversa a la de los empleados. Por una parte, la mayor proporción de mujeres $(66.5 \%)$, sólo alcanza el más bajo nivel de escolaridad. Esa característica se repite independientemente de los grupos de edad.

c) Análisis de la relación entre la fecundidad y el grado de escolaridad en la ciudad de México, según la edad y la posición social. El objetivo de este análisis es saber hasta dónde ejerce su acción la posición social de los jefes de familia, en el comportamiento de la relación entre la 
fecundidad y el grado de escolaridad en la ciudad de México. En otras palabras se pretende indagar qué matiz adquiere la relación una vez que se controla la posición social de los jefes de familia y la edad de las madres.

Estas interrogantes conducen a formular una vez más otro ejercicio de estandarización. Recuérdese que al igual que en los casos anteriores, para poder llevarlo a efecto, hay que tener presente que no exista interacción entre la posición social de los jefes de familia y la escolaridad, es decir, que para todos los grupos de edad y para todas las posiciones sociales, el efecto de la escolaridad en la fecundidad se tiene que dar en el mismo sentido.

Pasemos ahora a efectuar la estandarización. Se hizo una primera representación gráfica (véase la gráfica 2), en donde aparecen en forma conjunta, los diferentes grupos de posición social de los jefes de familia y el número medio de hijos vivos, según la edad y el nivel de escolaridad de las madres. Al observar la evolución que siguen las curvas, se aprecian diversos comportamientos. De manera concreta, para el grupo de edad 15-19 y 20-24 años, los empleados aparecen como la posición social en donde el número medio de hijos nacidos vivos es mayor, al menos en los dos primeros niveles de escolaridad. Luego, en los otros grupos de edad, la curva cae, lo que indica que el valor representado es menor. Situaciones similares se encuentran para los otros conjuntos de posición social. Así, mientras para un determinado grupo de edades la curva denota los valores más bajos del número medio de hijos nacidos vivos, luego ocupa una posición intermedia, e incluso una más alta. (Véase al respecto cómo cambia la posición de la curva que representa a los empleadores y autónomos no manuales en los diferentes grupos de edad, o en su defecto la de los autónomos manuales y trabajadores manuales).

La trayectoria gráfica que acabamos de describir, indica que el efecto de la escolaridad sobre la fecundidad es diferencial para cada uno de los grupos de posición social, acontecimiento que impide proceder a efectuar la estandarización, pues la condición de que no exista interacción entre la posición social y la escolaridad no está dada. Por otra parte, la secuencia que siguen las curvas, conduce a pensar que la variable posición social juega un papel más importante que el que le hubiéramos podido atribuir, es decir, se supone que dicha variable, más que un papel de control, desempeña uno de tipo predictivo.

Se procedió entonces a representar el número medio de hijos nacidos vivos, según la edad de las madres y el nivel de escolaridad para cada uno de los grupos de posición social. (Véase la gráfica 3). Como se puede observar, dentro de cada uno de los grupos de posición social, el efecto de la escolaridad en la fecundidad se da en el mismo sentido, independientemente de la edad. Obsérvese que, aun cuando las curvas no desciendan siempre con la misma intensidad - lo que las hace variar en su configuración- lo más interesante es que el descenso en el número medio de hi- 


\section{Gráfica 2}

Ciudad de México: Número Medio de hijos Nacidos vivos SEgứN

LA POSICIÓN SOCIAL DE LOS JEFES DE FAMILIA Y EL GRADO DE ESCOLARIDAD $Y$ EDAD DE LAS MADRES CASADAS $Y$ CONVIVIENTES, 1970

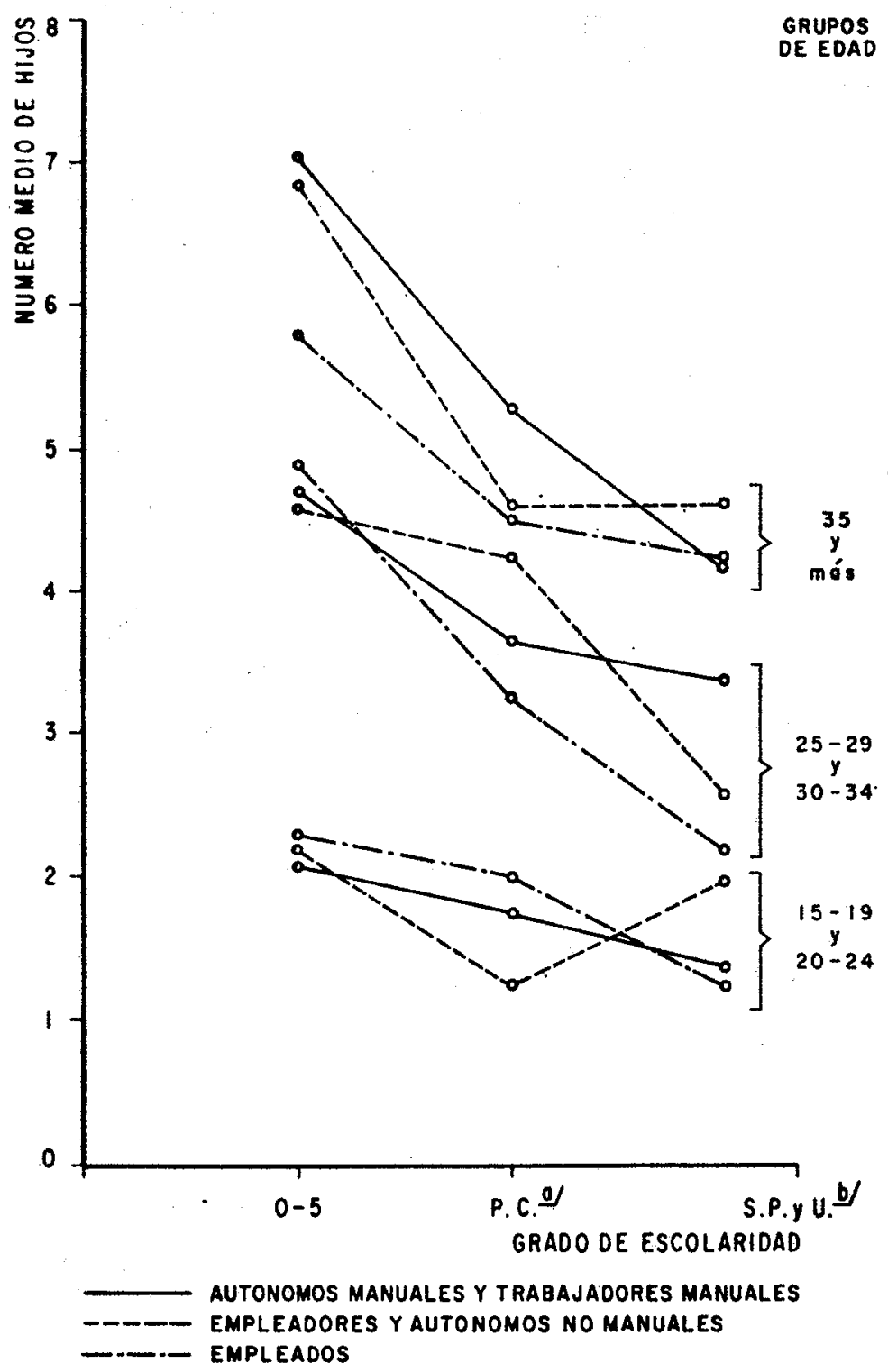

a) Primaria completo b/ Secundario, preporotorio y universidad 
jos, a medida que el nivel de escolaridad aumenta, se manifiesta para todos los grupos de edad, dentro de cada una de las posiciones sociales. Bajo estas condiciones se puede pasar a realizar la estandarización, aunque sea preciso efectuarla por separado para cada uno de los grupos de posición social. En el cuadro 13 se aprecian las "paridades medias" reales y estandarizadas, según el nivel de escolaridad, en cada uno de los grupos de posición social.

\section{Gráfica 3}

Ciudad de México: Número Medio de hijos Nacidos vivos SEgúN LA POSICIÓN SOCIAL DE LOS JEFES DE FAMILIA Y EL GRADO DE ESCOLARIDAD Y EDAD DE LAS MADRES CASADAS

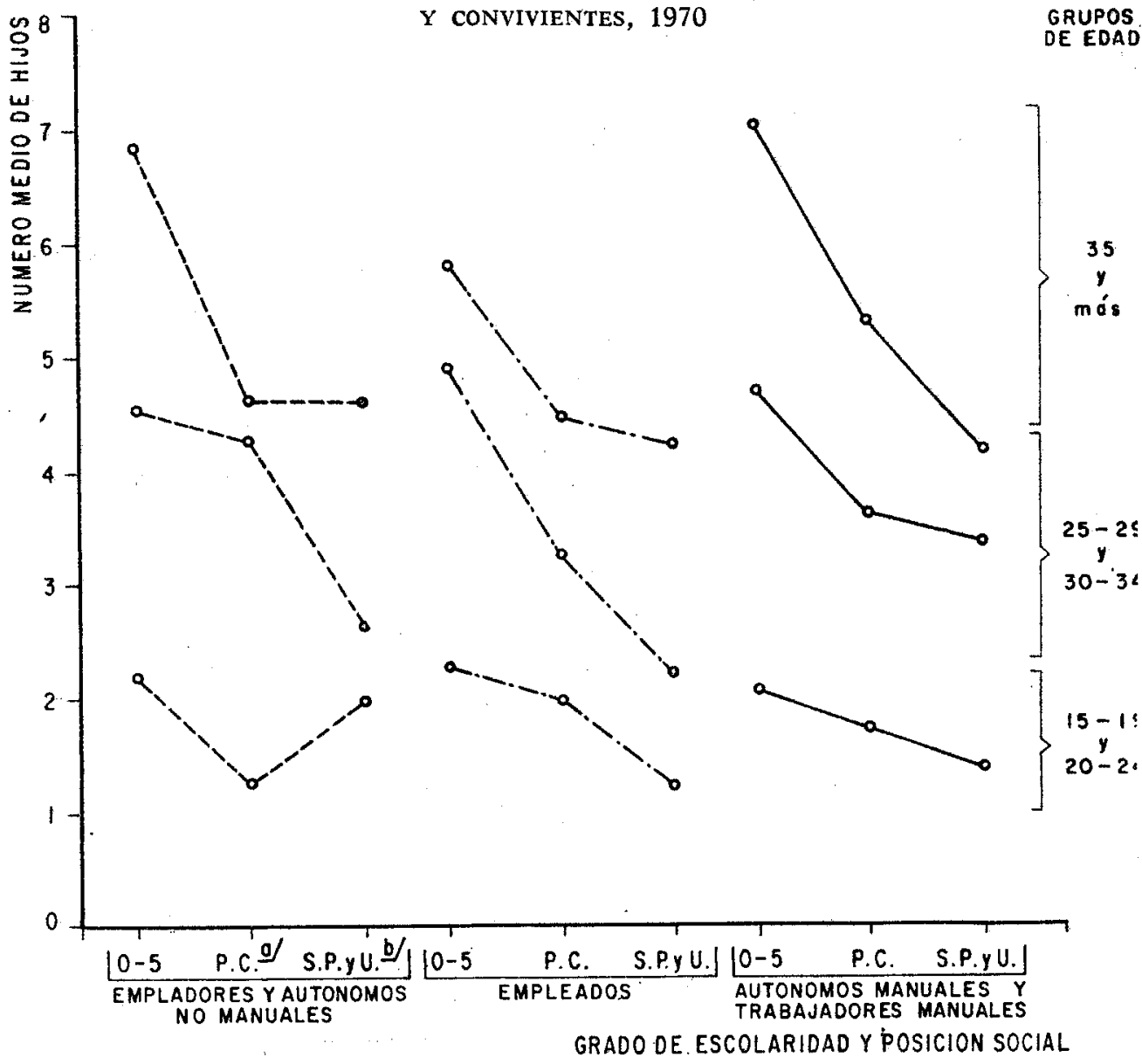

a) Primaria completa

b. Secundaria, preporatoria y universidad 


\section{Cuadro 13}

Crudad de MÉxico: Paridad MEdia REAL Y ESTANDARIzAda SEGÚN LA POSICIÓN SOCIAL Y EL GRADO DE ESCOLARIDAD

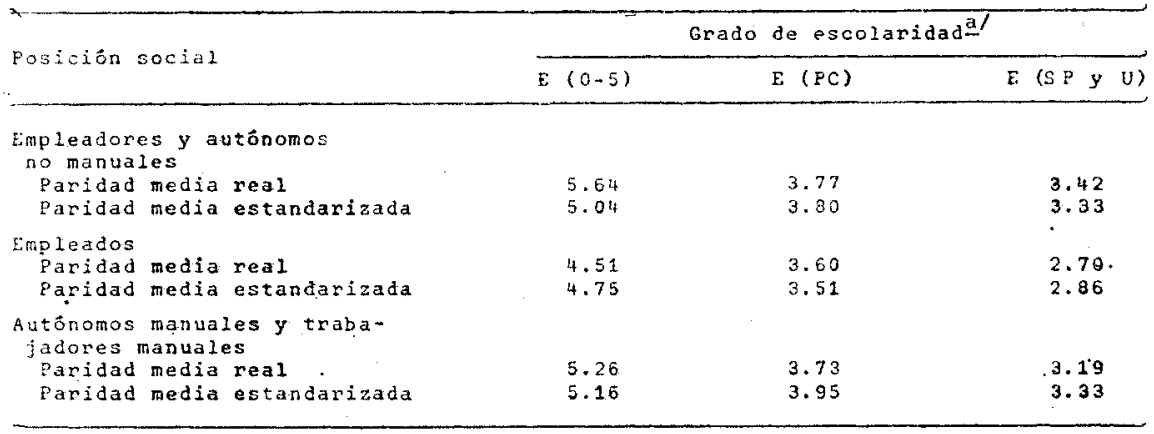

a $\mathrm{E}(0-5)$ : hasta cinco años de escolaridad; E (PC): primaria completa; E (SP y U): secundaria, preparatoria y universidad.

Se puede observar que los valores de las paridades reales no presentan mayor variación en relación a los valores de las paridades medias estandarizadas. Estos cambios sensibles, producto de haber adoptado como estructura tipo la del total de la población entrevistada casada y conviviente, indican que en la ciudad de México el efecto de la estructura por edad en la paridad media, una vez que se ha introducido alguna referencia a la estratificación social y, en consecuencia, se han clasificado las mujeres de acuerdo a esa referencia, no es muy considerable.

Ya antes se había llegado a la misma conclusión, al efectuar cálculos similares sin tener en cuenta la estratificación social. Sin embargo, las ligeras diferencias observadas varían dentro de cada uno de los grupos de posición social, lo cual refleja que el efecto de la estructura por edad, aunque no sea muy grande, es diferencial. Aquí sólo se comentan los casos más significativos. De manera concreta, dentro de los empleadores y autónomos no manuales, para el nivel de 0-5 grados de escolaridad, es donde se observa mayor discrepancia. El valor 0.60 hijos menos que acusa la paridad media estandarizada, indica que este subgrupo posee una estructura por edad un tanto envejecida. El caso contrario se observa para los empleados dentro del mismo nivel de escolaridad, y para los autónomos manuales y los trabajadores manuales a nivel de la primaria completa. En ambos casos la estructura por edad es un tanto más joven que la del conjunto de la población.

En conclusión, se puede decir que una vez clasificadas las mujeres según grupos de posición social, su estructura por edad no difiere mayormente de la estructura por edad de la población total entrevistada casada y conviviente. De ahí que no se den mayores cambios entre las paridades 
medias reales y las paridades medias estandarizadas, y que en todo caso las ligeras variaciones observadas no son otra cosa que la resultante de haberle aplicado a la paridad media por edad y nivel de escolaridad una estructura por edad levemente más vieja o más joven que la propia.

Veamos ahora qué sucede con los efectos netos reales y estandarizados (véase el cuadro 14).

Cuadro 14

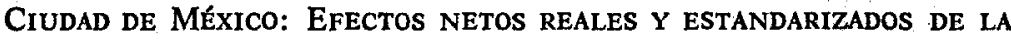
ESCOLARIDAD SOBRE LA PARIDAD MEDIA, SEGÚN LA POSICIÓN SOCIAL.

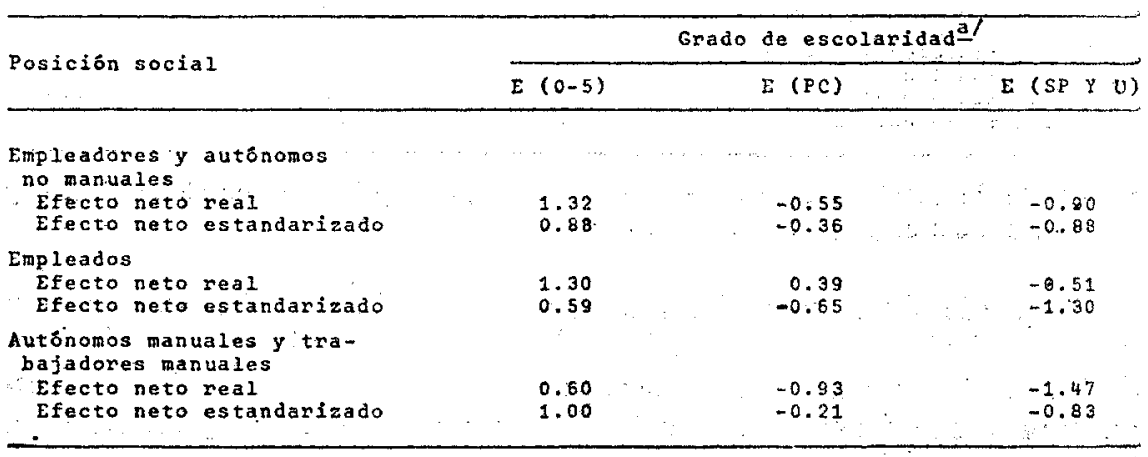

- Véase la nota del cuadro 13.

Dentro de los empleadores y autónomos no manuales, se puede apreciar que la diferencia más notable entre los efectos netos reales y los efectos netos estandarizados se da en el nivel de 0-5 grados de escolaridad. De 1.32 hijos más la paridad media pasa a 0.88 hijos más, lo cual indica que la diferencia de 0.44 hijos, es debido a la acción de la estructura por edad y que el hecho de haber alcanzado las mujeres ese nivel de escolaridad sólo las lleva a tener 0.88 hijos más que la paridad media dentro de ese grupo de posición social y no 1.32 hijos más, como lo expresa el efecto neto real.

En los dos niveles de escolaridad siguientes, la acción neta de la escolaridad en la fecundidad se deja sentir con mayor intensidad, aunque es inferior a la expresada por los efectos netos reales. Las diferencias menores que se aprecian para esos niveles de escolaridad, entre los valores de los efectos netos reales y los efectos netos estandarizados, señalan que la acción de la estructura por edad al interior de dichos niveles es más reducida que la ejercida en el primero de los casos.

Dentro del grupo de posición social correspondiente a los empleados, es donde el efecto de la escolaridad en la fecundidad se deja sentir con mayor intensidad. En dicho grupo, para todos los niveles de escolaridad, una vez eliminada la influencia de la estructura por edad, se observa que, 
por una parte, el número medio de hijos nacidos vivos disminuye en relación a la paridad media dentro de esa posición social, y por otra, es también donde las disminuciones alcanzan mayor valor. En otras palabras, las mujeres casadas y convivientes cuyos cónyuges son empleados tienen 0.59 hijos más que la paridad media si han alcanzado $0-5$ grados de escolaridad; 0.65 hijos menos si terminaron la primaria completa, y 1.30 hijos menos si lograron alguna posición dentro de la secundaria, la preparatoria o la universidad.

Dentro del conjunto formado por los autónomos manuales y los trabajadores manuales se encontró que la situación se invierte en relación a los empleados, y hasta cierto punto se asemeja bastante a la de los empleadores y autónomos no manuales, sobre todo en los dos últimos niveles de escolaridad. Una vez eliminada la influencia de la estructura por edad, el efecto neto de la escolaridad en la fecundidad es, dentro de este grupo de posición social, ligeramente menor que el apreciado en el caso de los empleadores y autónomos no manuales.

De manera concreta, se puede afirmar que las mujeres con 0-5 grados de escolaridad tienen 1 hijo más que la paridad media de ese grupo, y no 0.60 hijos más, lo cual indica que en la diferencia de 0.40 hijos menos que expresa el efecto neto real, pesan más otras influencias, entre ellas la de la estructura por edad, que la de la misma escolaridad. En los dos siguientes niveles de escolaridad también se aprecia que al eliminar la influencia de la estructura por edad, se reduce el efecto de la escolaridad; y que el hecho de alcanzar la primaria completa sólo conduce a tener 0.21 hijos menos, y una vez lograda alguna posición dentro de la secundaria, la preparatoria o la universidad, se tienen 0.83 hijos menos que la paridad media de ese grupo de posición social, pero no 1.47 hijos menos, como lo señala el efecto neto real.

En relación a este apartado se pueden anotar dos conclusiones. a) el efecto neto de la escolaridad en la paridad media comienza a observarse con mayor nitidez en los diferentes grupos de posición social, una vez que las mujeres superan la primaria completa, aunque en forma diferencial, en cada uno de dichos grupos $\mathrm{y} ; b$ ) que el valor más significativo que caracteriza al efecto neto estandarizado en todos los niveles de escolaridad dentro de los empleados, reafirma que es en ese grupo donde el efecto neto de la escolaridad se deja sentir con mayor intensidad en la fecundidad, y como tal, que no era un hecho fortuito el resultado encontrado desde el primer momento en que se hizo alusión a la posición social de los individuos. En otras palabras, el menor número de hijos nacidos vivos que caracterizó a los empleados a través de todo el análisis, se mantiene una vez aislada la influencia de la estructura por edad, y constituye un suceso definitivo y real.

Al observar en la ciudad de México el acontecimiento antes expuesto, es preciso encontrarle alguna explicación. Como es frecuente, siempre se piensa en algunos factores que puedan ejercer influencia. Entre otros, se 
citan la postergación del matrimonio, el mayor o menor uso de anticonceptivos, las aspiraciones de movilidad social, la influencia de la religión, el trabajo de la mujer, etc., los cuales por lo regular se asocian de una $\mathbf{u}$ otra manera con el tipo de ocupación y, por ende, con determinada posición social. Al menos por el momento, no se dispone de evidencias para probar ninguno de esos argumentos. En tal sentido, se prefiere más bien asociar la conducta reproductiva de los diferentes grupos de posición social en la ciudad de México con el comportamiento que le asigna Latapí a las clases sociales en relación a la educación (véase la parte I, pp. 12-13).

En términos de lo expresado por Latapí, es la clase media quien mejor se ha aprovechado de la educación en México, y si dentro de nuestra aproximación a una situación de estratificación piramidal, se puede considerar como clase media a los empleados, se encuentra justificación a su comportamiento reproductivo, en el sentido de asociar el contenido educacional de que han sido objeto, a su conducta reproductiva expresada en un número menor de hijos nacidos vivos. Claro está que no se debe entender su comportamiento reproductivo sólo bajo la influencia de la escolaridad. Parece apropiado este momento para recordar algunas de las alusiones a que se hizo mención respecto a los empleados, cuando se justificaba la agrupación de las posiciones sociales. En otras palabras, se hace mención de la propia naturaleza de sus condiciones, a los tipos de normas, valores, presiones, etc., a que se encuentran expuestos. De similar manera se puede razonar respecto a los otros dos grupos de posición social. Según Latapí, la clase alta no ha tenido en México mayor tradición cultural y, como tal, no se ha caracterizado por una elevada educación, mientras la clase baja, en términos generales, no se ha distinguido por recibir mayores oportunidades educativas.

Bajo esta óptica, parece sensato comprender por qué el efecto de la escolaridad en la fecundidad es menor dentro del grupo de los empleadores y autónomos no manuales, y aún inferior, en el caso de los autónomos manuales y de los trabajadores manuales. Al comparar la posición teórica de Latapí con lo expresado por los datos (véase el cuadro 12), se encontró que las formulaciones del mencionado autor encuentran apoyo, pues la proporción de mujeres dentro de cada uno de los grupos de posición social que alcanzan el nivel más elevado de escolaridad es de $43.2 \%$ en el caso de los empleadores y autónomos no manuales; de $61.9 \%$ para los empleados; y de $15.5 \%$ dentro de los autónomos manuales y los trabajadores manuales. Si se recuerda que el efecto neto de la escolaridad en la fecundidad se ejerce de manera principal una vez que se supera el nivel de la primaria completa, y por lógica aumenta a medida que se alcanza un nivel superior, sin ir muy lejos, los mismos datos son bastante explicativos por sí solos.

A manera de conclusión de este apartado, se puede decir que la asociación intensivamente más negativa que se esperaba encontrar en la ciudad de México entre la fecundidad y la escolaridad, resultó ser cierta, 
pero no de una forma tan nítida como se creía. Por otra parte, dicha asociación no adquirió la intensidad que se pronosticó, una vez que se hiciese alusión a la estratificación social de los individuos y se progresara en la escala de la estratificación social. Todo ello indica que si bien es cierto que el Distrito Federal es la entidad que mejor se ha beneficiado de la expansión del sistema educativo en México, ese sólo hecho parece reflejar la reducción en el número medio de hijos nacidos vivos, en relación al medio rural, a medida que se alcanza un nivel de escolaridad mayor, tanto en términos generales como al interior de los grupos de posición social. Pero no necesariamente se reproduce la relación intensiva, con frecuencia esperada, entre la fecundidad y el prestigio de la ocupación.

Aún con el peligro de incurrir en repeticiones, se piensa que es el momento de hacer alusión a algunas de las premisas esbozadas en la introducción de este trabajo. A lo que se quiere hacer mención es que el hecho de haber encontrado una caracterización muy particular para la relación entre la fecundidad y la escolaridad, vía posición social, en la ciudad de México, constituye la evidencia de que dicha relación no es algo tan sencillo como parece a primera vista. Esto debido a que tanto el proceso de reproducción como el nivel de escolaridad alcanzado no son variables libres, sino dependientes, que reflejan el juego de factores más amplios que los superan, engloban y determinan, es decir, que están de una $\mathbf{u}$ otra manera mediatizados por la realidad social. El comportamiento que puede caracterizar la relación a la que se ha hecho mención, en un momento dado, es aquel que obedece a su propia situación, y es comprensible sólo dentro de la totalidad donde se desenvuelve.

\section{CONCLUSIONES}

En esta última parte, se hace hincapié sobre los hechos más significativos, ya que a lo largo del trabajo se han venido haciendo explícitas algunas conclusiones. Estas, se dividen en dos grupos, tal como aparecen a continuación.

\section{En cuanto a la situación educativa:}

a) En términos generales, puede decirse que todos los indicadores utilizados en el análisis de la situación educativa para el período comprendido entre los años 1921 y 1970, mostraron que los servicios educativos han crecido en forma considerable, incluso en el medio rural;

b) Sin embargo, al no haber obedecido la expansión del sistema educativo a condiciones de planeación integral, se ha caracterizado por presentar una conformación en extremo piramidal, en donde subsisten desigualdades de todo tipo y, en donde el medio urbano y la ciudad de 
México conjuntamente con las regiones sociogeográficas de mayor desarrollo y los sectores medios y altos de los grupos de estratificación social, se han venido aprovechando de los mejores beneficios;

c) Para ilustrar las diferencias antes mencionadas, baste con recordar que el analfabetismo es diferencial a nivel de sexos y siempre mayor en las áreas rurales, así como también que la demanda potencial, la matrícula escola primaria, el movimiento y aprovechamiento de los alumnos, la proporción de escuelas primarias, el personal docente, etc., constituyen indicadores en donde siempre el contexto urbano y la ciudad de México, presentan una posición de primacía en relación al medio rural.

\section{En cuanto a la relación entre la fecundidad y la escolaridad:}

a) $\mathrm{Al}$ igual que en muchas partes en donde se ha examinado la mencionada relación, en la ciudad de México también se hizo evidente la asociación negativa entre la fecundidad y el grado de escolaridad;

b) El número medio de hijos nacidos vivos en la ciudad de México es de 4.20. En relación al medio rural mexicano (5.29), se observa una diferencia de 1.09 hijos menos en la ciudad capital. También ocurre en dicha ciudad que las diferencias observadas en cuanto al número medio de hijos, tanto entre los que reciben el más bajo y el más alto nivel de escolaridad, como también entre los niveles intermedios, son más significativas que en el medio rural;

c) En términos generales, una vez alejada la influencia de la estructura por edad, se encontró que el efecto neto de la escolaridad en el nivel de la fecundidad se comienza a ejercer cuando se alcanza la primaria completa. Como para la ciudad de México, en el primer análisis, se tuvo la oportunidad de conservar desglosadas la primaria completa, la secundaria y la preparatoria-universidad, se pudo apreciar que el efecto de la escolaridad en la fecundidad se incrementa a medida que el nivel de escolaridad es mayor y que su incidencia es más fuerte cuando se pasa de la secundaria a la preparatoria-universidad.

A título de comparación, Zambrano Lupi $(7,1977)$, encontró que en el medio rural mexicano, el umbral donde el efecto neto de la escolaridad sobre la fecundidad comienza a ejercer su influencia también está dado por el momento en que se alcanza la primaria completa. Sin embargo, dicho efecto es menor en el contexto rural que en la ciudad de México;

d) Introducida alguna referencia a la posición social de los individuos y eliminada la influencia de la estructura por edad, también se constató que el efecto de la escolaridad en el nivel de la fecundidad es mayor entre los empleados, que dentro de las otras posiciones sociales consideradas.

En el caso del medio rural mexicano, Zambrano Lupi (1977), al in- 
troducir la posición social (vista en función del tipo de ocupación de los cónyuges de las entrevistadas), encontró que al eliminar la influencia de la estructura por edad, el efecto neto de la escolaridad en la fecundidad se comienza a dejar sentir dentro de las ocupaciones agrícolas y tradicionales propias del medio estrictamente rural, cuando se alcanza el nivel de 6 y más grados de escolaridad. En las ocupaciones ni agrícolas ni tradicionales, propias del medio semi urbano, dicho efecto, aunque de una forma muy sutil, comienza a ejercer su influencia desde el nivel más bajo de escolaridad, aumentando progresivamente a medida que dicha escolaridad es mayor. Todo parece indicar que el contexto donde se realiza la ocupación es mucho más significativo en relación al nivel de la fecundidad, que la propia naturaleza de las ocupaciones;

e) Por último, en la ciudad de México, la hipótesis comúnmente esbozada que liga negativamente el nivel de la fecundidad con el prestigio de la ocupación no fue del todo evidente. Resulta que no es dentro del grupo de más alta posición social donde el número medio de hijos nacídos vivos es menor, sino en los grupos de posición social media, de manera concreta dentro de los empleados. Esta última circunstancia nos muestra una realidad que muchas veces se descuida: La relación entre la fecundidad y la escolaridad adopta por necesidad el comportamiento del contexto donde se desarrolla, al margen de que las evidencias empiricas puedan asignarle una caracterización general.

\section{ANEXO}

1. Algunas consideraciones de la encuesta sobre migración interna, estructura ocupacional y movilidad social en el área metropolitana de la ciudad de México

Esta encuesta, dados los objetivos del estudio sobre Migración y desigualdad social en la ciudad de México, se realizó en dos etapas y comprendió en total tres encuestas. La primera etapa (Fase $A$ ), consistió en la aplicación de una cédula colectiva de tipo censal a una muestra representativa de la población del área metropolitana de la ciudad de México. La segunda etapa (Fase $B$ ), consistió en la aplicación de una cédula de carácter individual y detallado, a una submuestra de hombres y mujeres de la población anteriormente mencionada.

El trabajo de campo de la primera etapa (Fase $A$ ), y de la cual se obtuvo la información para la realización del cuarto inciso del presente estudio, se inició en octubre de 1969, luego de un año de análisis de todo lo relacionado con la investigación en forma conjunta.

Cecilia Galli $(18,1977$, p. 17), sintetiza las características generales de la Fase $A$, de la manera siguiente: Para la primera etapa del trabajo (Fase $A$ ), se obtuvo una muestra estratificada bietápica, represen- 
tativa del área metropolitana de la ciudad de México de 2500 viviendas, en las cuales se aplicó la cédula de entrevista censal a todos los habitantes del hogar, resultando una población total de 13000 personas. De ese total 1453 mujeres resultaron ser casadas o convivientes. Se recolectó información sobre las siguientes variables: población, migración, estructura ocupacional y movilidad social.

Por último se advierte que no se entró en detalles respecto a la segunda etapa (Fase $B$ ), debido a que no se utilizó dicha información para los efectos de este trabajo.

\section{Breve síntesis de los ejercicios de estandarización}

La estandarización o tipificación, como también suele llamársele, según Carleton $(19,1970$, p. 49), es "el método clásico usado en demografía como substituto del experimento de laboratorio contratado de las ciencias experimentales". La estandarización $(19,1970$, p. 47), "efectúa una manipulación estadística y artificial de las variables que hasta cierto punto alteran la situación que se está analizando, si se trata de varibles interdependientes". Es el caso, por ejemplo, del nivel de la fecundidad y la estructura por edad de la población. La estandarización en este caso proporciona una técnica para estimar cuál sería el nivel de la fecundidad en cada nivel de escolaridad, bajo el supuesto de que cada grupo tuviera la misma estructura por edad de la población tipo (en este caso, la estructura por edad del total de la población de mujeres entrevistadas casadas y convivientes).

\section{Aclaraciones adicionales}

1. El efecto del grado de escolaridad sobre la paridad media se calculó restando el valor de la paridad media total a cada valor de la paridad media según grado de escolaridad.

2. La interpretación de esos valores hay que entenderla como una desviación (positiva o negativa), del valor de la paridad media total.

3. Para el cálculo de las paridades medias estandarizadas, se utiliza una fórmula del tipo siguiente:

Supóngase el caso del nivel 0-3 grados de escolaridad.

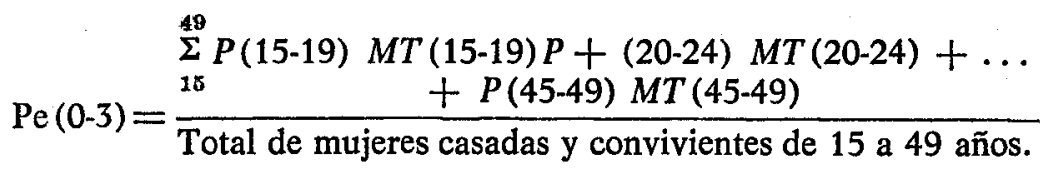


En donde: $\mathrm{Pe}(0-3)=$ Paridad media estandarizada para el nivel de 0-3 años de escolaridad.

$\boldsymbol{P}=$ paridad media.

$M T=$ total de mujeres en cada grupo de edad.

La sumatoria contiene el número de hijos nacidos, estandarizados por edad, los cuales al dividirse por el total de madres casadas y convivientes, dan como resultado la paridad media estandarizada para determinado nivel de escolaridad.

4. La paridad media estandarizada por grado de escolaridad resulta de dividir el número de hijos nacidos vivos entre el total de las madres casadas y convivientes.

5. La paridad media total estandarizada se obtiene al multiplicar las paridades, según grado de escolaridad, estandarizadas, por el total de mujeres que hay en cada nivel de escolaridad.

6. En todas las estandarizaciones, para el cálculo de las paridades medias estandarizadas, siempre se ponderó por el total de mujeres casadas y convivientes que cubrió la encuesta.

\section{REFERENCIAS BIBLIOGRAFICAS}

1. Miró, Carmen y Walter Mertens, Influencia de algunas variables intermedias en el nivel y en los diferenciales de fecundidad urbana y rural en América Latina, Santiago de Chile, Celade, 1969.

2. Muñoz, Humberto, Orlandina de Oliveira y Claudio Stern, Migración y desigualdad social en la ciudad de México, México, Instituto de Investigaciones Sociales de la UNAM y El Colegio de México, 1977.

3. Muñoz Izquierdo, Carlos, "Evaluación del desarrollo educativo en México (1958-70) y factores que to han determinado", Revista del Centro de Estudios Educativos, Vol. III, Núm. 3, 1973.

4. Latapí, Pablo, Mitos y verdades de la educación mexicana 1971-72, México, Centro de Estudios Educativos, 1973.

5. Medellín, Rodrigo: "Educación, estructura de clases y cambio social", Revista del Centro de Estudios Educativos, Vol. III, Núm. 3, 1973.

6. Timur, Serim, Variables demográficas correlacionadas con la edad de la mujer: Fecundidad, edad al matrimonio y familia, México, Unión Internacional para el Estudio Científico de la Población, 1977.

7. Zambrano Lupi, Jorge H., "La relación entre la fecundidad y el grado de escolaridad en el medio rural mexicano y en la ciudad de México", México, El Colegio de México, 1977.

8. Singer, Paul, Economía politica de la urbanización, México, Siglo XXI Editores, 1975.

9. Quijano, Aníbal, "Urbanización y tendencias de cambio en la sociedad rural latinoamericana", en: Desarrollo urbano y regional en América 
Latina, Selección de Luis Unikel y Andrés Necochea, Fondo de Cultura Económica, México, 1975.

10. Kaplan, Marcos, La ciudad latinoamericana como factor de transmisión de poder socioeconómico y político hacia el exterior durante el período contemporáneo, en Martha Schteingart (Comp.), Urbanización y dependencia en América Latina, Buenos Aires, Ediciones Siap, 1973.

11. Goldani, Ana María, "Evaluación de los datos de la población total y de la población inmigrante captados por la encuesta", en Humberto Muñoz y otros (Comps.), Migración y desigualdad social en la ciudad de México, México, Instituto de Investigaciones Sociales, UNAM, y El Colegio de México, 1977.

12. Pullum, T. W. Standarization, Technical Bulletin No 3, International Statistical Institute, 1977.

13. García, Brígida y Orlandina de Oliveira, "Características socioeconómicas de las unidades domésticas del Distrito Federal", DEMografía y EcoNomía, Vol. XIII, Núm. 1, 1979.

14. Becker, Gary, "An Economic Analysis of Fertility", en Demographic and Economic Change in Developed Countries, Princeton, Princeton University Press, 1960.

15. Leibenstein, Harvey, “The Economic Theory of Fertility Decline", The Quarterly Journal of Economics, Vol. LXXXIX, No 1, febrero de 1975.

16. Leibenstein, Harvey, La teoría económica de la fecundidad: investigaciones, problemas y consideraciones, México, Unión Internacional para el Estudio Científico de la Población, 1977.

17. Bultato, Rodolfo, Relaciones entre el valor y el costo de los hijos y la fecundidad, una evidencia a través de la cultura, México, Unión Internacional para el Estudio Científico de la Población, 1977.

18. Galli, Cecilia, "La metodología de la encuesta", en Humberto Muñoz y otros, op. cit.

19. Carleton, Robert, Aspectos metodológicos y sociológicos de la fecundidad humana, Santiago de Chile, CELADE, 1970. 